\title{
Review Article \\ On Fault Detection in Linear Discrete-Time, Periodic, and Sampled-Data Systems
}

\author{
P. Zhang and S. X. Ding \\ Institute for Automatic Control and Complex Systems, Faculty of Engineering, University of Duisburg-Essen, \\ Bismarckstrasse 81 BB, 47057 Duisburg, Germany \\ Correspondence should be addressed to S. X. Ding, steven.ding@uni-due.de
}

Received 30 April 2007; Accepted 4 October 2007

Recommended by Kemin Zhou

This paper gives a review of some standard fault-detection (FD) problem formulations in discrete linear time-invariant systems and the available solutions. Based on it, recent development of FD in periodic systems and sampled-data systems is reviewed and presented. The focus in this paper is on the robustness and sensitivity issues in designing model-based FD systems.

Copyright (c) 2008 P. Zhang and S. X. Ding. This is an open access article distributed under the Creative Commons Attribution License, which permits unrestricted use, distribution, and reproduction in any medium, provided the original work is properly cited.

\section{INTRODUCTION}

With the increasing requirements of modern complex control systems on safety and reliability, model-based fault detection and isolation (FDI) technology has attracted remarkable attention during the last three decades [1-6]. In major industrial sectors, it has become an important supporting technology and is replacing the traditional hardware redundancy technique in part or totally. As a standard functional module, FDI systems are increasingly integrated in modern technical systems and provide valuable information for condition-based predictive maintenance, higher-level fault tolerant control, and plant-wide production optimization.

Though closely related to the development of control and filtering theory, there are several distinct features of the model-based FDI problems that justify the efforts made in this field. To evaluate the performance of an FDI system in practice, miss alarm rate, false alarm rate, and detection delay are the most important criteria that decide the acceptance of the methods. It is widely accepted that these functional requirements can be reformulated as a multi-objective problem. Enhancing the robustness of the FDI system to unknown disturbances and modeling errors is an essential objective. However, alone the robustness does not guarantee a good FDI performance. The sensitivity of the FDI system to faults should be simultaneously improved. To find the best compromise between the robustness and the sensitivity is thus the central problem in model-based FDI. This is the first difference of FDI problems from control and standard filtering problems, where the focus is put on disturbance attenuation. Bearing this in mind, full-decoupling problem and optimal design of FDI systems have been studied [3-6] and different types of indices have been introduced to describe the sensitivity to the faults. Secondly, for the purpose of FDI, a fault indicating signal, called residual, needs not only to be generated, but also to be evaluated and, based on it, a decision for the existence, location, and size of the faults needs to be made. Therefore, an FDI procedure includes residual generation and residual evaluation. An integrated design of these two parts is needed to guarantee the optimal FDI performance [7].

In this paper, we will first give a review of some standard fault detection (FD) problem formulations in discretetime systems and the available solutions. There are two types of discrete-time model-based FD systems: the parity space and the observer-based ones. The former is, in its original form, specially dedicated to the discrete-time systems [8], while the latter is analogous to the continuous-time systems and its development shares the same essentials with the continuous-time systems. Perhaps for this reason, besides the early research activity on the parity space approaches, only few studies have been specifically devoted to the FD problems in discrete-time systems. Recently, the intensive research on networked control systems (NCS) and embedded systems 
considerably stimulates the study on periodic, sampled-data systems [9]. The integration of data communication networks into control systems introduces natural periodic behavior in the system dynamics and the sampling effect is understood not only in view of the behavior of $\mathrm{A} / \mathrm{D}$ and $\mathrm{D} / \mathrm{A}$ converters but also in the context of data transmission among the subsystems. It can be observed that the recent studies on FD in periodic and sampled-data systems are mainly based on the discrete-time model-based FD methods. It is this fact that motivates us to give an overview of some standard FD methods for discrete-time systems and, based on it, to review and present some recent results on FD in periodic and sampled-data systems. Bearing in mind that fault isolation problems can be principally reformulated as a robust fault detection problem $[4,5]$, our focus in this paper is on the robustness issues in designing model-based FD systems.

The paper is organized as follows. In Section 2, we review FD methods for discrete-time systems and address some important relations between different methods. Section 3 is devoted to FD in discrete-time periodic systems. In Section 4, FD in sampled-data systems is addressed.

Throughout this paper, standard notations of robust control theory, for instance those used in [10], are adopted. We will use $\underline{\sigma}(X), \bar{\sigma}(X)$ to denote the minimum and maximum singular values of matrix $X$, respectively and $\sigma_{i}(X)$ to denote any singular value of $X$ that satisfies $\underline{\sigma}(X) \leq \sigma_{i}(X) \leq$ $\bar{\sigma}(X)$. $\|x\|_{E}$ denotes the Euclidean norm of vector $x,\|\xi\|_{2}$ the $l_{2}$-norm of discrete-time signal $\xi$ or the $\mathcal{L}_{2}$-norm of continuous-time signal $\xi$, $\|\xi\|_{2,[a, b)}$ the 2 norm of $\xi$ over the interval $[a, b)$, and $\|G\|_{\infty}$ the $H_{\infty}$-norm of transfer function matrix $G$. The superscript $T$ denotes the transpose of matrices and the superscript $*$ denotes the adjoint of operators. $\mathcal{R} \mathscr{H}_{\infty}$ stands for the subspace that consists of all proper and real rational stable transfer function matrices. In this paper, we call a state-space model $(A, B, C, D)$ regular, if it is detectable and has no invariant zeros on the unit circle and no unobservable modes at the origin.

\section{FD OF DISCRETE LTI SYSTEMS}

Linear time-invariant (LTI) systems are the simplest class of systems. Although the handling of FD problems in discrete LTI systems can often be done along the well-established framework of FD schemes for continuos LTI systems, study on FD in discrete LTI systems is of primary importance from the following three aspects:

(i) it gives insight and often motivates extensions to more complex systems like periodic and sampled-data systems addressed in the subsequent sections;

(ii) there are some methods that have been developed specially for discrete LTI systems;

(iii) due to its practical form for the direct online implementation, the discrete-time system form is often favored in the applications.

In this section, basic ideas and solution procedures of advanced FD methods for discrete LTI systems, divided into three groups, will be reviewed: (i) parity space approaches, which are specific for discrete LTI systems and will be dealt to some details;

(ii) the parametrization of observer-based FD systems and postfilter design schemes;

(iii) fault detection filter schemes, which are mostly studied and closely related to robust control theory.

Thanks to the well-known relationships between the technical features of the discrete- and continuous-time systems, many well-established FD schemes for continuous LTI systems can be directly applied to the latter two FD schemes. For this reason, we will restrict ourselves to some representative methods and give a brief view of the analog application of the methods for continuous-time systems to the discrete FD systems. Another focus in this section is on the comparison and interpretation of the FD methods.

\subsection{System models and problem formulation}

Suppose that the discrete LTI systems are described by

$$
\Sigma_{\mathrm{LTI}}:\left\{\begin{array}{l}
x(k+1)=A x(k)+B u(k)+E_{d} d(k)+E_{f} f(k), \\
y(k)=C x(k)+D u(k)+F_{d} d(k)+F_{f} f(k),
\end{array}\right.
$$

where $x \in \mathcal{R}^{n}$ is the state vector, $u \in \mathcal{R}^{p}$ the vector of control inputs, $y \in \mathcal{R}^{m}$ the vector of process outputs, $d \in \mathcal{R}^{n_{d}}$ the vector of unknown disturbances, and $f \in \mathcal{R}^{n_{f}}$ the vector of faults to be detected, $A, B, E_{d}, E_{f}, C, D, F_{d}$, and $F_{f}$ are known constant matrices of appropriate dimensions. In the frequency domain, system $\Sigma_{\text {LTI }}$ can be equivalently described by

$$
y(z)=G_{u}(z) u(z)+G_{d}(z) d(z)+G_{f}(z) f(z),
$$

where $G_{u}(z), G_{d}(z)$, and $G_{f}(z)$ denote, respectively, the transfer function matrices from $u, d$, and $f$ to $y$.

Although the design of a model-based FD systems mainly consists of three tasks: (a) residual generation, (b) residual evaluation, (c) threshold determination, major research attention has been focused on the residual generation with the following issues.

(i) Full decoupling problem, which deals with the design of a residual generator, so that the residual signal $r$ satisfies

$$
\begin{aligned}
\forall u, d, \lim _{k \rightarrow \infty} r(k)=0 & \text { if } f=0, \\
r(k) \neq 0 & \text { if } f_{i}(k) \neq 0, i=1, \ldots, n_{f} .
\end{aligned}
$$

If a full decoupling is realized, then the residual evaluation reduces to detect the nonzeroness of the residual signal.

(ii) Optimal FD problem, which is to design the residual generator so that the residual signal $r$ is as small as possible if $f=0$ and deviates from 0 as much as possible if $f_{i}(k) \neq 0, i=1, \ldots, n_{f}$.

Considering that in the fault-free case the residual signal $r$ would, due to the existence of $d$, differ from zero, evaluation 
of the size of $r$ is necessary in order to distinguish the influence of the faults from that of the disturbances. In this paper, the norm-based evaluation of the residual signal, denoted by $J=\|r\|$ and, based on it, threshold determination satisfying

$$
J \text { th }=\sup _{f=0, d}\|r\|
$$

will be briefly reviewed.

\subsection{Parity space approach}

The parity space approach is based on the so-called parity relation. Let $s$ be an integer denoting the length of a moving time window. The output of system (1) over the moving window $[k-s, k]$ can be expressed by the initial state $x(k-s)$, the stacked control input vector $u_{k, s}$, the stacked disturbance vector $d_{k, s}$, and the stacked fault vector $f_{k, s}$ as

$$
y_{k, s}=H_{o, s} x(k-s)+H_{u, s} u_{k, s}+H_{d, s} d_{k, s}+H_{f, s} f_{k, s}
$$

where

$$
\begin{aligned}
\xi_{k, s} & =\left[\begin{array}{c}
\xi(k-s) \\
\xi(k-s+1) \\
\vdots \\
\xi(k)
\end{array}\right] \quad \text { with } \xi \text { standing for } y, u, d, f, \\
H_{o, s} & =\left[\begin{array}{c}
C \\
C A \\
\vdots \\
C A^{s}
\end{array}\right], \quad H_{u, s}=\left[\begin{array}{cccc}
D & O & \cdots & O \\
C B & D & \ddots & \vdots \\
\vdots & \ddots & \ddots & O \\
C A^{s-1} B & \cdots & C B & D
\end{array}\right],
\end{aligned}
$$

$H_{d, s}$ and $H_{f, s}$ are constructed similarly as $H_{u, s}$ and can be achieved by replacing $B, D$, respectively, by $E_{d}, F_{d}$ and $E_{f}, F_{f}$. To satisfy the requirement on the residual signal, a residual generator can be constructed as

$$
r(k)=v_{s}\left(y_{k, s}-H_{u, s} u_{k, s}\right)
$$

where a design parameter $v_{s}$ called parity vector is introduced to modulate the residual dynamics and improve the sensitivity of the residual to the faults and the robustness to the disturbances and the initial state. Usually, $v_{s} H_{o, s}=0$ is required to eliminate the influence of the initial state and the past input signals (before the time instant $k-s$ ).

If the existence condition

$$
\operatorname{rank}\left[\begin{array}{lll}
H_{o, s} & H_{d, s} & H_{f, s}
\end{array}\right]>\operatorname{rank}\left[\begin{array}{ll}
H_{o, s} & H_{d, s}
\end{array}\right]
$$

is satisfied, then a full decoupling from both the initial state and the disturbances can be achieved by solving

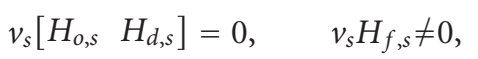

for $v_{s}$, that is, $v_{s}$ lies in the intersection between the left null space of $\left[\begin{array}{ll}H_{o, s} & H_{d, s}\end{array}\right]$ and the image space of $H_{f, s}$. If a full de- coupling is not achievable or not desired, the FD problem is often formulated as to solve the optimization problem

$$
\begin{aligned}
\max _{v_{s}, v_{s} H_{o, s}=0} J_{\mathrm{PS}}\left(v_{s}\right) & =\max _{v_{s}, v_{s} H_{o, s}=0} \frac{\sup _{d_{k, s}=0, f_{k, s} \neq 0}\left(r^{T}(k) r(k) / f_{k, s}^{T} f_{k, s}\right)}{\sup _{f_{k, s}=0, d_{k, s} \neq 0}\left(r^{T}(k) r(k) / d_{k, s}^{T} d_{k, s}\right)} \\
& =\max _{v_{s}, v_{s} H_{o, s}=0} \frac{v_{s} H_{f, s} H_{f, s}^{T} v_{s}^{T}}{v_{s} H_{d, s} H_{d, s}^{T} v_{s}^{T}}
\end{aligned}
$$

whose solution can be obtained by solving a generalized eigenvalue-eigenvector problem [11].

\section{Solution to optimization problem (10)}

Let $N_{\text {basis }}$ denote the basis of the left null space of $H_{o, s}$. Assume that $\lambda_{\max }$ and $p_{s, \max }$ are the maximal generalized eigenvalue and the corresponding eigenvector to the generalized eigenvalue-eigenvector problem

$$
p_{s, \max }\left(N_{\text {basis }} H_{f, s} H_{f, s}^{T} N_{\text {basis }}^{T}-\lambda_{\max } N_{\text {basis }} H_{d, s} H_{d, s}^{T} N_{\text {basis }}^{T}\right)=0,
$$

then optimization problem (10) is solved by

$$
v_{s}=p_{s, \max } N_{\text {basis }} \text {. }
$$

It is pointed out in [12] that the solutions of a full decoupling or (10) are achieved at the cost of (considerably) reduced fault detectability. This can be immediately seen with a look at the dynamics of the residual signal

$$
r(k)=v_{s}\left(y_{k, s}-H_{u, s} u_{k, s}\right)=v_{s}\left(H_{f, s} f_{k, s}+H_{d, s} d_{k, s}\right)
$$

which shows that the influence of the fault expressed by $v_{s} H_{f, s}$ is structurally reduced to a minimum, that is, $\operatorname{rank}\left(v_{s} H_{f, s}\right)=1$. Reference [12] proposed the use of a parity matrix $V_{s}$,

$$
r(k)=V_{s}\left(y_{k, s}-H_{u, s} u_{k, s}\right)=V_{s}\left(H_{f, s} f_{k, s}+H_{d, s} d_{k, s}\right)
$$

instead of a parity vector aiming at enhancing the influence of the faults on the residual signal. To this end, the following optimization problems are formulated as

$$
\begin{aligned}
& \max _{V_{s}, V_{s} H_{o, s}}=0 J_{\mathrm{PS}, \infty / \infty}\left(V_{s}\right) \\
&= \max _{V_{s}, V_{s} H_{o, s}=0} \frac{\sup _{d_{k, s}=0, f_{k, s} \neq 0}\left(r^{T}(k) r(k) / f_{k, s}^{T} f_{k, s}\right)}{\sup _{f_{k, s}=0, d_{k, s} \neq 0}\left(r^{T}(k) r(k) / d_{k, s}^{T} d_{k, s}\right)} \\
&=\max _{V_{s}, V_{s} H_{o, s}=0} \frac{\bar{\sigma}^{2}\left(V_{s} H_{f, s}\right)}{\bar{\sigma}^{2}\left(V_{s} H_{d, s}\right)}, \\
& \max _{V_{s}, V_{s} H_{o, s}=}=0 J_{\mathrm{PS},-/ \infty}\left(V_{s}\right) \\
&=\max _{V_{s}, V_{s} H_{o, s}=0} \frac{\inf _{d_{k, s}=0, f_{k, s} \neq 0}\left(r^{T}(k) r(k) / f_{k, s}^{T} f_{k, s}\right)}{\sup _{f_{k, s}=0, d_{k, s} \neq 0}\left(r^{T}(k) r(k) / d_{k, s}^{T} d_{k, s}\right)} \\
&=\max _{V_{s}, V_{s} H_{o, s}=0} \frac{\sigma^{2}\left(V_{s} H_{f, s}\right)}{\bar{\sigma}^{2}\left(V_{s} H_{d, s}\right)}, \\
& \max _{V_{s}, V_{s} H_{o, s}=0} J_{\mathrm{PS}, i / \infty}\left(V_{s}\right) \max _{V_{s}, V_{s} H_{o, s}=0} \frac{\sigma_{i}^{2}\left(V_{s} H_{f, s}\right)}{\bar{\sigma}^{2}\left(V_{s} H_{d, s}\right)} .
\end{aligned}
$$


The difference in optimization problems (15)-(16) consists in that the former considers the maximal influence of the faults on the residual amplitude, while the latter considers the minimal influence. Optimization problem (17) is a generalization of (15)-(16) and takes into account the fault sensitivity in different directions. The achievable optimal performance index of optimization problem (15) is the same with that of (10). At the end of this subsection, we will show that the solution of (17) would lead to maximizing the fault detectability in the context of a tradeoff between false alarm rate and fault detectability.

\section{Solution to optimization problems (15), (16), and (17)}

A solution to optimization problem (17) that also solves (15)-(16) simultaneously is given in [12] as follows, which is derived based on the observation that for any matrices $X_{1}$ and $X_{2}$ of compatible dimensions,

$$
\begin{aligned}
\bar{\sigma}\left(X_{1} X_{2}\right) & \leq \bar{\sigma}\left(X_{1}\right) \bar{\sigma}\left(X_{2}\right), \\
\underline{\sigma}\left(X_{1} X_{2}\right) & \leq \bar{\sigma}\left(X_{1}\right) \underline{\sigma}\left(X_{2}\right), \\
\sigma_{i}\left(X_{1} X_{2}\right) & \leq \bar{\sigma}\left(X_{1}\right) \sigma_{i}\left(X_{2}\right) .
\end{aligned}
$$

Assume that there is the following singular value decomposition (SVD):

$$
N_{\text {basis }} H_{d, s}=U\left[\begin{array}{ll}
S & O
\end{array}\right] V^{T},
$$

where $U$ and $V$ are unitary matrices, $S=\operatorname{diag}\left\{\sigma_{1}, \ldots, \sigma_{\gamma}\right\}$, then optimization problems (15), (16), and (17) are solved by

$$
V_{s}=\bar{P}_{s} S^{-1} U^{T} N_{\text {basis }},
$$

where $\bar{P}_{s}$ is any unitary matrix of compatible dimensions.

Note that the solutions to the above optimization problems are not unique. For instance, an alternative optimal solution for problem (16) is

$$
V_{s}=\bar{P}_{s} P N_{\text {basis }},
$$

where $P$ is the left inverse of $N_{\text {basis }} H_{f, s}$. On the other side, only solution (20) solves (15), (16), and (17) simultaneously. For this reason, (20) is called unified parity space solution.

To detect the faults successfully, the generated residual signal should be further evaluated. For a residual signal generated by means of the parity space approach, the Euclidean norm defined by

$$
J=\|r(k)\|_{E}=\sqrt{r^{T}(k) r(k)}
$$

is a reasonable evaluation function. It follows from (14) and (4) that the corresponding threshold is determined by

$$
J \text { th }=\sup _{f=0, d}\|r(k)\|_{E}=\bar{\sigma}\left(V_{s} H_{d, s}\right) \max \left\|d_{k, s}\right\|_{E} .
$$

Based on the decision logic

$$
\|r(k)\|_{E} \leq J \text { th } \Longrightarrow \text { fault-free, otherwise faulty, }
$$

a decision for the occurrence of a fault can be finally made.
In practice, false alarm rate and miss detection rate are two important technical features for the performance evaluation of a fault detection system. Below, we will introduce these two concepts in the context of the norm-based residual evaluation (22) and briefly compare the above-presented parity space solutions.

$J$ th setting under a given false alarm rate. Consider (23) and denote the upper bound of $\left\|d_{k, s}\right\|_{E}$ by $\delta_{d}$. In the context of norm-based evaluation, the objective of $J_{\text {th }}$ setting is to ensure that any disturbance whose size is not larger than the tolerant limit should not cause an alarm. To express the strongest disturbance that is allowed without causing a false alarm in relation to $\delta_{d}$, we define false alarm rate (FAR) as

$$
\mathrm{FAR}=1-\frac{\alpha}{\delta_{d}}, \quad 0 \leq \alpha \leq \delta_{d} \Longrightarrow 0 \leq 1-\frac{\alpha}{\delta_{d}} \leq 1,
$$

that is, those disturbances whose size is not larger than $\alpha=$ $(1-\mathrm{FAR}) \delta_{d}$ should not cause an alarm. Suppose that the allowable FAR is now given. It is straightforward that the threshold should be set as

$$
J \text { th }=\alpha \bar{\sigma}\left(V_{s} H_{d, s}\right)=(1-\mathrm{FAR}) \delta_{d} \bar{\sigma}\left(V_{s} H_{d, s}\right) .
$$

Note that in the norm-based residual evaluation, $J$ th is often set as $\delta_{d} \bar{\sigma}\left(V_{s} H_{d, s}\right)$ which leads to a zero FAR but may result in a very conservative $J$ th setting.

To express the miss detection rate (MDR), we introduce the set of detectable faults. Note that a fault can be detected if and only if

$$
\|r\|_{E}>J \text { th } \Longleftrightarrow\left\|V_{s}\left(H_{d, s} d_{k, s}+H_{f, s} f_{k, s}\right)\right\|_{E}>J \text { th. }
$$

Hence, the set of detectable faults (SDF) $\Omega_{\mathrm{DE}}\left(V_{s}, J\right.$ th, $\left.d\right)$ is defined as follows: given $V_{s}$ and $J$ th

$$
\Omega_{\mathrm{DE}}\left(V_{s}, J \text { th, } d\right)=\left\{f_{k, s} \mid(27) \text { is satisfied }\right\} .
$$

Given $J$ th, a parity space matrix $V_{s, \text { opt }}$ delivers a residual signal with the lowest MDR if

$$
\forall V_{s}, \Omega_{\mathrm{DE}}\left(V_{s}, J \text { th }, d\right) \subseteq \Omega_{\mathrm{DE}}\left(V_{s, \mathrm{opt}}, J \text { th }, d\right),
$$

that is, $\Omega_{\mathrm{DE}}\left(V_{s, \mathrm{opt}}, J\right.$ th, $\left.d\right)$ includes the largest number of detectable faults, which is equivalent with the lowest MDR.

The subsequent comparison study is done in the context of maximizing SDF (i.e., minimizing $M D R$ ) under a given FAR.

Note that (27) can be, according to (19), rewritten into

$$
\begin{aligned}
\left\|V_{s}\left(H_{d, s} d_{k, s}+H_{f, s} f_{k, s}\right)\right\|_{E} \\
\quad>(1-\mathrm{FAR}) \delta_{d} \bar{\sigma}\left(V_{s} H_{d, s}\right) \\
\quad \Longleftrightarrow\left\|Q\left[\begin{array}{ll}
I & O
\end{array}\right] V^{T} d_{k, s}+Q S^{-1} U^{T} N_{\text {basis }} H_{f, s} f_{k, s}\right\|_{E} \\
\quad>(1-\mathrm{FAR}) \delta_{d} \bar{\sigma}\left(Q\left[\begin{array}{ll}
I & O
\end{array}\right] V^{T}\right)
\end{aligned}
$$

by setting $V_{s}=Q S^{-1} U^{T} N_{\text {basis, }}$ for some $Q$.

It turns out that $\forall Q \neq 0,(30)$ holds only if

$$
\left\|\left[\begin{array}{ll}
I & O
\end{array}\right] V^{T} d_{k, s}+S^{-1} U^{T} N_{\text {basis }} H_{f, s} f_{k, s}\right\|_{E}>(1-\mathrm{FAR}) \delta_{d} .
$$


It means that a parity matrix that ensures (31) would provide a maximal SDF. Note that the unified parity space solution (20) delivers exactly (31). Thus, the unified parity space solution maximizes SDF (i.e., minimizes MDR) under a given FAR.

For comparison, denote the SVD of $N_{\text {basis }} H_{f, s}$ by

$$
N_{\text {basis }} H_{f, s}=U_{f}\left[\begin{array}{ll}
S_{f} & O
\end{array}\right] V_{f}^{T}
$$

Then the vector-valued solution (12) to optimization problem (10) and the matrix-valued solution (21) to optimization problem (16) can be, respectively, rewritten into

$$
\begin{gathered}
v_{s}=p_{s} S^{-1} U^{T} N_{\text {basis }}, \\
p_{s}\left(S^{-1} U^{T} U_{f} S_{f}^{2} U_{f}^{T} U S^{-1}-\lambda_{\max } I\right)=0, \\
V_{s}=\left(\bar{P}_{s} S_{f}^{-1} U_{f}^{T} U S\right) S^{-1} U^{T} N_{\text {basis. }} .
\end{gathered}
$$

Since, generally, $p_{s}, \bar{P}_{s} S_{f}^{-1} U_{f}^{T} U S$ are not unitary matrices, we have finally

$$
\begin{array}{r}
\Omega_{\mathrm{DE}}\left(V_{s}, J \text { th }, d\right) \subset \Omega_{\mathrm{DE}}\left(V_{s, \mathrm{opt}}, J \text { th }, d\right), \quad V_{s}=p_{s, \max } N_{\text {basis }}, \\
V_{s, \text { opt }}=\bar{P}_{s} S^{-1} U^{T} N_{\text {basis }}, \\
\Omega_{\mathrm{DE}}\left(V_{s}, J \text { th }, d\right) \subset \Omega_{\mathrm{DE}}\left(V_{s, \mathrm{opt}}, J \text { th }, d\right), \quad V_{s}=\bar{P}_{s} P N_{\text {basis }}, \\
V_{s, \text { opt }}=\bar{P}_{s} S^{-1} U^{T} N_{\text {basis }} .
\end{array}
$$

With the following remarks we would like to conclude this subsection.

(i) Parity-space-based FD system design is characterized by the simple mathematical handling. It only deals with matrix- and vector-valued operations. This fact attracts attention from the industry for the application of parity-space-based methods.

(ii) There is a one-to-one relationship between the parityspace approach and the observer-based approach that allows the design of an observer-based residual generator based on a given parity vector $[13,14]$. Based on this result, a strategy called parity-space design, observer-based implementation has been developed, which makes use of the computational advantage of parity-space approaches for the FD system design (selection of a parity vector or matrix) and then realizes the solution in the observer form to ensure a numerically stable and less consuming online computation. This strategy has been successfully used in the sensorfault detection in vehicles [15].

(iii) In the parity-space approaches, a high order $s$ will improve the optimal performance index $J_{\mathrm{PS}}$ but, on the other side, increase the online computational effort [16]. By introducing a low-order IIR (infinite impulse response) filter, the performance of the parityrelation-based residual generator can be much improved without significant increase of the order of the parity relation [17]. Similar effect can be achieved by the closed-loop-observer-based implementation, as pointed out by [18]. (iv) The algebraic form of the parity-space-based FD system allows a statistic test and norm-based residual evaluation and threshold determination [19]. It may well bridge the statistical methods [1] and the observer-based methods.

(v) In the framework of parity-space-based FD system design, system dynamic features like transmission zeros, zeros in the right half plane (RHP), and so forth are not taken into account. This may cause trouble at the online implementation. Also for this reason, we are of the opinion that the strategy of parity space design, observer-based implementation would be helpful to solve this problem.

\subsection{Parametrization of FD systems and postfilter design}

Observer-based FD system design for continuous LTI systems has been widely studied in the literature [3-6]. In this and the next subsections, the analog form of those known results will be briefly reviewed. Attention will be paid to the comparison study when it is special for discrete LTI systems.

Let $\left(\widehat{M}_{u}(z), \widehat{N}_{u}(z)\right)$ be a left coprime factorization pair of $G_{u}(z)$, that is, $G_{u}(z)=\widehat{M}_{u}^{-1}(z) \hat{N}_{u}(z), \widehat{M}_{u}(z), \widehat{N}_{u}(z) \in \mathcal{R} \mathscr{H}_{\infty}$ [10]. In [20], a parametrization of all LTI residual generators for system (1) described by

$$
r(z)=R(z)\left(\widehat{M}_{u}(z) y(z)-\widehat{N}_{u}(z) u(z)\right)
$$

is presented, where $R(z) \in \mathcal{R} \mathscr{H}_{\infty}$ is the so-called postfilter that is arbitrarily selectable. Suppose that $G_{u}(z)=$ $(A, B, C, D)$ is a detectable state space realization of $G_{u}(z)$. Then $\widehat{M}_{u}(z), \widehat{N}_{u}(z)$ can be computed as follows [10]:

$$
\begin{aligned}
\widehat{M}_{u}(z) & =I-C(z I-A+L C)^{-1} L \\
\widehat{N}_{u}(z) & =D+C(z I-A+L C)^{-1}(B-L D) .
\end{aligned}
$$

It is now a well-known result that

(i) $\widehat{M}_{u}(z) y(z)-\widehat{N}_{u}(z) u(z)=y(z)-\hat{y}(z)$, where $\hat{y}(z)$ is the output estimation delivered by a full-order observer;

(ii) given $L_{1}$ and $L_{2}$, there exists an $\mathcal{R} \mathscr{H}_{\infty}$-invertible postfilter $Q(z)=I+C\left(z I-A+L_{1} C\right)^{-1}\left(L_{2}-L_{1}\right)$ so that

$$
\begin{aligned}
& \widehat{M}_{u, L_{1}}(z) y(z)-\widehat{N}_{u, L_{1}}(z) u(z) \\
& \quad=Q(z)\left(\widehat{M}_{u, L_{2}}(z) y(z)-\widehat{N}_{u, L_{2}}(z) u(z)\right),
\end{aligned}
$$

where $\left(\widehat{M}_{u, L_{1}}(z), \widehat{N}_{u, L_{1}}(z)\right)$ and $\left(\widehat{M}_{u, L_{2}}(z), \widehat{N}_{u, L_{2}}(z)\right)$ are the left coprime factorization pair of $G_{u}(z)$, which are computed according to (36) with $L=L_{1}$ and $L=L_{2}$, respectively.

(iii) all LTI residual generators can be expressed by a series connection of a full-order observer and a postfilter, and are therefore called observer-based residual generators. Moreover, the selection of the postfilter can be done independent of the observer design. 
Due to the latter fact, we concentrate in this subsection on the selection of $R(z)$. The dynamics of a residual generator (35) is governed by

$$
r(z)=R(z) \widehat{M}_{u}(z)\left(G_{d}(z) d(z)+G_{f}(z) f(z)\right) .
$$

The full decoupling problem is to find the postfilter $R(z)$ so that

$$
R(z) \widehat{M}_{u}(z) G_{d}(z)=0, \quad R(z) \widehat{M}_{u}(z) G_{f}(z) \neq 0 .
$$

If the $l_{2}$-norm of the residual signal is used as evaluation function, then the optimal FD problem is formulated as optimization problems

$$
\begin{gathered}
\sup _{R(z) \in \mathcal{R} \mathcal{H}_{\infty}} J_{\mathrm{FRE}, \infty / \infty}(R)=\sup _{R(z) \in \mathcal{R} \mathcal{H}_{\infty}} \frac{\left\|R(z) \widehat{M}_{u}(z) G_{f}(z)\right\|_{\infty}}{\left\|R(z) \widehat{M}_{u}(z) G_{d}(z)\right\|_{\infty}}, \\
\sup _{R(z) \in \mathcal{R} \mathcal{H}_{\infty}} J_{\mathrm{FRE},-\infty \infty}(R)=\sup _{R(z) \in \mathcal{R} \mathcal{H}_{\infty}} \frac{\left\|R(z) \widehat{M}_{u}(z) G_{f}(z)\right\|_{-}}{\left\|R(z) \widehat{M}_{u}(z) G_{d}(z)\right\|_{\infty}}, \\
\sup _{R(z) \in \mathcal{R} \mathcal{H}_{\infty}} J_{\mathrm{FRE}, i / \infty}(R) \\
=\sup _{R(z) \in \mathcal{R} \mathcal{H}_{\infty}} \frac{\sigma_{i}\left(R\left(e^{j \omega}\right) \widehat{M}_{u}\left(e^{j \omega}\right) G_{f}\left(e^{j \omega}\right)\right)}{\left\|R(z) \widehat{M}_{u}(z) G_{d}(z)\right\|_{\infty}} \\
\sup _{R(z) \in \mathcal{R} \mathcal{H}_{\infty}^{1 \times m}}^{J_{\mathrm{FRE}, 2 / 2}(R)} \\
=\sup _{R(z) \in \mathcal{R} \mathcal{H}_{\infty}^{1 \times m}}\left(\int_{0}^{2 \pi} R\left(e^{j \omega}\right) \widehat{M}_{u}\left(e^{j \omega}\right) G_{f}\left(e^{j \omega}\right) G_{f}^{*}\left(e^{j \omega}\right)\right. \\
\times \widehat{M}_{u}^{*}\left(e^{j \omega}\right) R^{*}\left(e^{j \omega}\right) d \omega / \int_{0}^{2 \pi} R\left(e^{j \omega}\right) \\
\times \widehat{M}_{u}\left(e^{j \omega}\right) G_{d}\left(e^{j \omega}\right) G_{d}^{*}\left(e^{j \omega}\right) \widehat{M}_{u}^{*}\left(e^{j \omega}\right) \\
\left.\times R^{*}\left(e^{j \omega}\right) d \omega\right),
\end{gathered}
$$

where $\sigma_{i}\left(R\left(e^{j \omega}\right) \widehat{M}_{u}\left(e^{j \omega}\right) G_{f}\left(e^{j \omega}\right)\right)$ represents the fault sensitivity at different levels at the frequency $\omega$,

$$
\begin{aligned}
\left\|R(z) \widehat{M}_{u}(z) G_{f}(z)\right\|_{-} & =\inf _{\omega}\left(\underline{\sigma}\left(R\left(e^{j \omega}\right) \widehat{M}_{u}\left(e^{j \omega}\right) G_{f}\left(e^{j \omega}\right)\right)\right) \\
& =\inf _{\omega, i}\left(\sigma_{i}\left(R\left(e^{j \omega}\right) \widehat{M}_{u}\left(e^{j \omega}\right) G_{f}\left(e^{j \omega}\right)\right)\right)
\end{aligned}
$$

though not a norm, it is interpreted as the worst-case fault sensitivity. Optimization problems (40), (41), and (43) are often called the $H_{\infty} / H_{\infty}, H_{-} / H_{\infty}$, and $H_{2} / H_{2}$ optimization, respectively.

The ratio-type performance index given in (40) and (43) is the first one that was introduced for the FD purpose [11, 21]. Currently, the index of the form

$$
\left\|R(z) \widehat{M}_{u}(z) G_{d}(z)\right\|<\gamma, \quad\left\|R(z) \widehat{M}_{u}(z) G_{f}(z)\right\|>\beta
$$

becomes more popular, where $\gamma, \beta$ are some constants. The FD system design is often formulated as maximizing $\beta$ under a given $\gamma$. The third index type is often met in the robust control theory and formulated as

$$
J_{f-d}=\alpha_{f}\left\|R(z) \widehat{M}_{u}(z) G_{f}(z)\right\|-\alpha_{d}\left\|R(z) \widehat{M}_{u}(z) G_{d}(z)\right\|,
$$

where $\alpha_{f}, \alpha_{d}>0$ are some given constants. The FD system design is then achieved by maximizing $J_{f-d}$. In [22], it has been demonstrated that the above three types indices are equivalent in a certain sense. With this fact in mind, in this paper we only consider optimizations under ratio-type indices (40)-(43).

\section{Solution to optimization problems (40)-(42)}

Let $\widehat{M}_{u}(z) G_{d}(z)=G_{d o}(z) G_{d i}(z)$ be a co-inner-outer factorization of $\widehat{M}_{u}(z) G_{d}(z)$ [10], where $G_{d o}(z)$ is the $\mathcal{R} \mathscr{H}_{\infty}$-leftinvertible co-outer, $G_{d i}(z)$ is the co-inner containing all the right half complex plane zeros of $\widehat{M}_{u}(z) G_{d}(z)$ and satisfying $G_{d i}(z) G_{d i}^{*}(z)=I$. Based on the relations

$$
\begin{aligned}
\left\|G_{1}(z) G_{2}(z)\right\|_{\infty} & \leq\left\|G_{1}(z)\right\|_{\infty}\left\|G_{2}(z)\right\|_{\infty}, \\
\left\|G_{1}(z) G_{2}(z)\right\|_{-} & \leq\left\|G_{1}(z)\right\|_{\infty}\left\|G_{2}(z)\right\|_{-} \\
\sigma_{i}\left(G_{1}\left(e^{j \omega}\right) G_{2}\left(e^{j \omega}\right)\right) & \leq\left\|G_{1}(z)\right\|_{\infty} \sigma_{i}\left(G_{2}\left(e^{j \omega}\right)\right), \quad \forall i,
\end{aligned}
$$

it has been proven in $[23,24]$, similar to the results for continuous LTI systems given in [22] and recently in [25], that optimization problems (40)-(42) are solved simultaneously by

$$
R(z)=G_{d o}^{-1}(z)
$$

For this reason, (48) is called unified solution.

Another solution to optimization problem $(41)$, if $G_{f}(z)$ is $\mathcal{R} \mathcal{L}_{\infty}$-left-invertible, is

$$
R(z)=G_{f o}^{-1}(z)
$$

where $G_{f_{o}}(z)$ is the co-outer of $\widehat{M}_{u}(z) G_{f}(z)$.

The main purpose of the co-inner-outer factorization is to separate the nonminimum phase zeros so that the rest part of $\widehat{M}_{u}(z) G_{d}(z)$ or $\widehat{M}_{u}(z) G_{f}(z)$ is $\mathcal{R} \mathscr{H}_{\infty}$-left invertible. Note that the co-inner-outer factorization is not unique. Therefore, the optimal postfilter $R(z)$ is also not unique [26].

\section{Solution to optimization problem (43)}

The optimal solution to optimization problem (43) is a frequency selector as follows [27]:

$$
R(z)=f_{\omega_{0}}(z) p(z), \quad J_{\mathrm{FRE}, 2 / 2, \mathrm{opt}}=\sup _{\omega} \lambda_{\max }(\omega),
$$

where $f_{\omega_{0}}(z)$ is an ideal frequency-selective filter with the selective frequency at $\omega_{0}$, which satisfies

$$
\begin{aligned}
& f_{\omega_{0}}\left(e^{j \omega}\right) q\left(e^{j \omega}\right)=0, \quad \omega \neq \omega_{0} \\
& \int_{0}^{2 \pi} f_{\omega_{0}}\left(e^{j \omega}\right) q\left(e^{j \omega}\right) q^{*}\left(e^{j \omega}\right) f_{\omega_{0}}^{*}\left(e^{j \omega}\right) d \omega \\
& \quad=q\left(e^{j \omega_{0}}\right) q^{*}\left(e^{j \omega_{0}}\right), \quad \forall q^{T}(z) \in \mathcal{R} \mathscr{H}_{2},
\end{aligned}
$$


$\lambda_{\max }(\omega), p\left(e^{j \omega}\right)$ are, respectively, the maximal generalized eigenvalue and corresponding eigenvector of the following generalized eigenvalue-eigenvector problem:

$$
\begin{gathered}
p\left(e^{j \omega}\right)\left(\lambda_{\max }(\omega) \widehat{M}_{u}\left(e^{j \omega}\right) G_{d}\left(e^{j \omega}\right) G_{d}^{*}\left(e^{j \omega}\right) \widehat{M}_{u}^{*}\left(e^{j \omega}\right)\right. \\
\left.-\widehat{M}_{u}\left(e^{j \omega}\right) G_{f}\left(e^{j \omega}\right) G_{f}^{*}\left(e^{j \omega}\right) \widehat{M}_{u}^{*}\left(e^{j \omega}\right)\right)=0
\end{gathered}
$$

and $\omega_{0}$ is the frequency at which $\lambda_{\max }(\omega)$ achieves its maximum, that is,

$$
\lambda_{\max }\left(\omega_{0}\right)=\sup _{\omega} \lambda_{\max }(\omega)
$$

In practice, usually a narrow bandpass filter is implemented as frequency selector. From the viewpoint of FAR and MDR, the frequency selector may cause loss of fault sensitivity and restrict the application of the $\mathrm{H}_{2} / \mathrm{H}_{2}$ optimal residual generator.

Recently, [27] reported a very interesting result on the relationship between the parity-space vector and the solution to optimization problem (43). It has been shown that

$$
\lim _{s \rightarrow \infty} \min _{v_{s}} \frac{v_{s} H_{f, s} H_{f, s}^{T} v_{s}^{T}}{v_{s} H_{d, s} H_{d, s}^{T} v_{s}^{T}}=\lambda_{\max }\left(\omega_{\mathrm{opt}}\right)
$$

and $\lim _{s \rightarrow \infty} v_{s}$ correspond to a bandpass filter. This result not only reveals the physical interpretation of the standard optimal selection of parity vectors but also provides us with an efficient tool to approximate the optimal solution to optimization problem (43). Moreover, based on it, advanced parity-space approaches using wavelet transform have been proposed [28, 29].

As to the residual evaluation and threshold determination, the $l_{2}$-norm is the mostly used evaluation function, which leads to

$$
\begin{aligned}
J & =\|r\|_{2}, \\
J \text { th } & =\sup _{f=0, d}\|r\|_{2}=\left\|R(z) \widehat{M}_{u}(z) G_{d}(z)\right\|_{\infty} \max \|d\|_{2} .
\end{aligned}
$$

In case of applying the unified solution (48), we have

$$
J \text { th }=\sup _{f=0, d}\|r\|_{2}=\max \|d\|_{2} .
$$

Analog with the results in [30] and the discussion in the last subsection, it can be proven that the unified solution (48) minimizes MDR under a given FAR, where MDR and FAR are defined in the context of the norm-based residual evaluation.

\section{4. fault detection filter design}

fault detection filter (FDF) is a special kind of observer-based FD systems (35) with a constant postfilter and constructed as

$$
\begin{aligned}
\hat{x}(k+1) & =A \hat{x}(k)+B u(k)+L(y(k)-\hat{y}(k)), \\
r(k) & =W(y(k)-\hat{y}(k)), \\
\hat{y}(k) & =C \hat{x}(k)+D u(k),
\end{aligned}
$$

where the observer gain matrix $L$ and the weighting matrix $W$ are design parameters. Due to its state space expression and close relation to the observer design, FDF study receives most research attention. The dynamics of residual generator (57) is governed by

$$
\begin{aligned}
r(z) & =G_{r d}(z) d(z)+G_{r f}(z) f(z), \\
G_{r d}(z) & =W\left(F_{d}+C(z I-A+L C)^{-1}\left(E_{d}-L F_{d}\right)\right), \\
G_{r f}(z) & =W\left(F_{f}+C(z I-A+L C)^{-1}\left(E_{f}-L F_{f}\right)\right) .
\end{aligned}
$$

In the FDF design, the full-decoupling problem is to design $L$ and $W$ such that

$$
G_{r d}(z)=0, \quad G_{r f}(z) \neq 0
$$

while the optimal FD problems are formulated so as to choose matrices $L, W$ that solve the optimization problem $[5,7,31]$

$$
\begin{aligned}
& \sup _{L, W} J_{\mathrm{OBS}, \infty / \infty}(L, W)=\sup _{L, W} \frac{\left\|G_{r f}(z)\right\|_{\infty}}{\left\|G_{r d}(z)\right\|_{\infty}}, \\
& \sup _{L, W} J_{\mathrm{OBS},-/ \infty}(L, W)=\sup _{L, W} \frac{\left\|G_{r f}(z)\right\|_{-}}{\left\|G_{r d}(z)\right\|_{\infty}}, \\
& \sup _{L, W} J_{\mathrm{OBS}, i / \infty}(L, W)=\sup _{L, W} \frac{\sigma_{i}\left(G_{r f}\left(e^{j \omega}\right)\right)}{\left\|G_{r d}(z)\right\|_{\infty}} .
\end{aligned}
$$

\section{Solution to optimization problems (60)-(62)}

Because the FDF (57) is a special case of (35), the optimal solutions to optimization problems (60)-(62) can be derived based on the state space realization of the optimal postfilter $R(z)$ given by (48), as shown in [24]. A unified optimal solution to optimization problems (60)-(62) is associated to a discrete-time algebraic Riccati system (DTARS) [24, 32]. Assume that $\left(A, E_{d}, C, F_{d}\right)$ is regular, then

$$
L=-L_{d}^{T}, \quad W=W_{d}
$$

solve optimization problems (60)-(62) simultaneously, where $W_{d}$ is the left inverse of a full column rank matrix $H_{d}$ satisfying $H_{d} H_{d}^{T}=C X_{d} C^{T}+F_{d} F_{d}^{T}$ and $\left(X_{d}, L_{d}\right)$ is the stabilizing solution to the DTARS

$$
\left[\begin{array}{cc}
A X_{d} A^{T}-X_{d}+E_{d} E_{d}^{T} & A X_{d} C^{T}+E_{d} F_{d}^{T} \\
C X_{d} A^{T}+F_{d} E_{d}^{T} & C X_{d} C^{T}+F_{d} F_{d}^{T}
\end{array}\right]\left[\begin{array}{c}
I \\
L_{d}
\end{array}\right]=0 .
$$

An alternative solution to optimization problem (61), if $\left(A, E_{f}, C, F_{f}\right)$ is regular, is given by

$$
L=-L_{f}^{T}, \quad W=W_{f}
$$

where $W_{f}$ is the left inverse of a full column rank matrix $H_{f}$ satisfying $H_{f} H_{f}^{T}=C X_{f} C^{T}+F_{f} F_{f}^{T}$ and $\left(X_{f}, L_{f}\right)$ is the stabilizing solution to the DTARS

$$
\left[\begin{array}{cc}
A X_{f} A^{T}-X_{f}+E_{f} E_{f}^{T} & A X_{f} C^{T}+E_{f} F_{f}^{T} \\
C X_{f} A^{T}+F_{f} E_{f}^{T} & C X_{f} C^{T}+F_{f} F_{f}^{T}
\end{array}\right]\left[\begin{array}{c}
I \\
L_{f}
\end{array}\right]=0 .
$$


Recently, application of LMI-technique (linear matrix inequality) to solve (60) and (61) for continuous LTI systems has been reported [33-36]. The core of those approaches consists in formulating (60) or (61) as a multiobjective optimization problem and solving them based on an iterative computation of two LMIs. It can be proven that these solutions can, in the ideal case, converge to the optimal solution (63). The extension of these results to the discrete FDF is straightforward and will not be discussed in this paper.

At the end of this subsection, we would like to introduce a very interesting result achieved by the comparison study between the well-known Kalman-filter-based residual generation [37] and the unified solution (63). Given system

$$
\begin{aligned}
x(k+1) & =A x(k)+B u(k)+E_{d} \zeta(k), \\
y(k) & =C x(k)+D u(k)+F_{d} \eta(k),
\end{aligned}
$$

where $\zeta(k), \eta(k)$ are independent zero-mean Gaussian white noise processes with $\operatorname{cov}\left(\zeta(k) \zeta^{T}(k)\right)=I, \operatorname{cov}\left(\eta(k) \eta^{T}(k)\right)=$ $I$, then a Kalman filter with

$$
\begin{array}{r}
\hat{x}(k+1 \mid k)=A \hat{x}(k \mid k-1)+B u(k)+L(y(k)-\hat{y}(k \mid k-1)), \\
\hat{y}(k \mid k-1)=C \hat{x}(k \mid k-1)+D u(k), \\
L=A P C^{T}\left(C P C^{T}+R\right)^{-1}, \quad R=F_{d} F_{d}^{T}(>0), \\
A P A^{T}-P-A P C^{T}\left(C P C^{T}+R\right)^{-1} C P A^{T}+E_{d} E_{d}^{T}=0, \quad P>0,
\end{array}
$$

delivers an innovation as residual signal, where $\hat{x}(k \mid k-1)$ is a prediction of $x(k)$ based on the data up to $k-1$. Comparing the above Kalman filter algorithm with the unified solution (63) makes it clear that both solutions are quite similar. Indeed, (67) can be brought into the general form of (1) by letting $d=\left[\begin{array}{ll}\zeta^{T} & \eta^{T}\end{array}\right]^{T}$ and, as a result, (71) can be regarded as a special case of (64). Remember that the Kalman filter delivers, in the context of statistic tests, a minimum MDR under a given FAR. In comparison, in the context of norm-based definition of MDR and FAR, the unified solution (63) provides us with the same result.

\section{FD OF DISCRETE-TIME LINEAR PERIODIC SYSTEMS}

In this section, we review some recent results on FD in discrete linear time periodic (LTP) systems. LTP is a special kind of linear time-varying systems described by

$$
\Sigma_{\mathrm{LTP}}:\left\{\begin{array}{l}
x(k+1) \\
\quad=A(k) x(k)+B(k) u(k)+E_{d}(k) d(k)+E_{f}(k) f(k), \\
y(k) \\
\quad=C(k) x(k)+D(k) u(k)+F_{d}(k) d(k)+F_{f}(k) f(k),
\end{array}\right.
$$

where $A(k), B(k), C(k), D(k), E_{d}(k), E_{f}(k), F_{d}(k)$, and $F_{f}(k)$ are known bounded and real periodic matrices of period $\theta$, that is, $\forall k, A(k+\theta)=A(k)$, and so forth. There is not only continuous interest and development of periodic control and filtering theory [38-42], but also increasing applications of periodic control in practice like helicopter vibration control, satellite attitude control as well as wind turbine.
The FD problem of periodic systems has been considered in [24, 32, 43-45]. Basically, there are two ways to handle the FD problem of LTP systems, as shown below.

\subsection{FD schemes based on lifted LTI reformulation}

It is well known that there is a strong correspondence between discrete LTP systems and discrete LTI systems [42]. Therefore, FD system design for the LTP system (72) can be carried out as follows:

(i) lift the LTP system (72) into a discrete LTI system,

(ii) design residual generator(s) based on the lifted LTI reformulation,

(iii) using either parallel residual generators or select the parameters of the residual generator to satisfy the causality condition.

Let $\Psi_{A(k)}\left(k_{1}, k_{0}\right)\left(k_{1} \geq k_{0}\right)$ denote the state transition matrix of LTP system (72)

$\Psi_{A(k)}\left(k_{1}, k_{0}\right)= \begin{cases}I & \text { if } k_{1}=k_{0}, \\ A\left(k_{1}-1\right) A\left(k_{1}-2\right) \cdots A\left(k_{0}\right) & \text { if } k_{1}>k_{0} .\end{cases}$

Periodic system (72) can be lifted into a discrete LTI system described by [42]

$$
\begin{aligned}
\tilde{x}_{\tau}((k+1) \theta+\tau)= & \widetilde{A}_{\tau} \tilde{x}_{\tau}(k \theta+\tau)+\widetilde{B}_{\tau} \tilde{u}_{\tau}(k \theta+\tau) \\
& +\widetilde{E}_{d, \tau} \tilde{d}_{\tau}(k \theta+\tau)+\widetilde{E}_{f, \tau} \tilde{f}_{\tau}(k \theta+\tau), \\
\tilde{y}_{\tau}(k \theta+\tau)= & \widetilde{C}_{\tau} \tilde{x}_{\tau}(k \theta+\tau)+\widetilde{D}_{\tau} \tilde{u}_{\tau}(k \theta+\tau) \\
& +\widetilde{F}_{d, \tau} \tilde{d}_{\tau}(k \theta+\tau)+\widetilde{F}_{f, \tau} \tilde{f}_{\tau}(k \theta+\tau),
\end{aligned}
$$

where $\tau$ is an integer between 0 and $\theta-1$ denoting the initial time, the state vector of the lifted system is $\tilde{x}_{\tau}(k \theta+\tau)=x(k \theta+$ $\tau), \tilde{\eta}_{\tau}$ with $\eta$ standing for $u, y, d, f$ is the augmented signal defined by

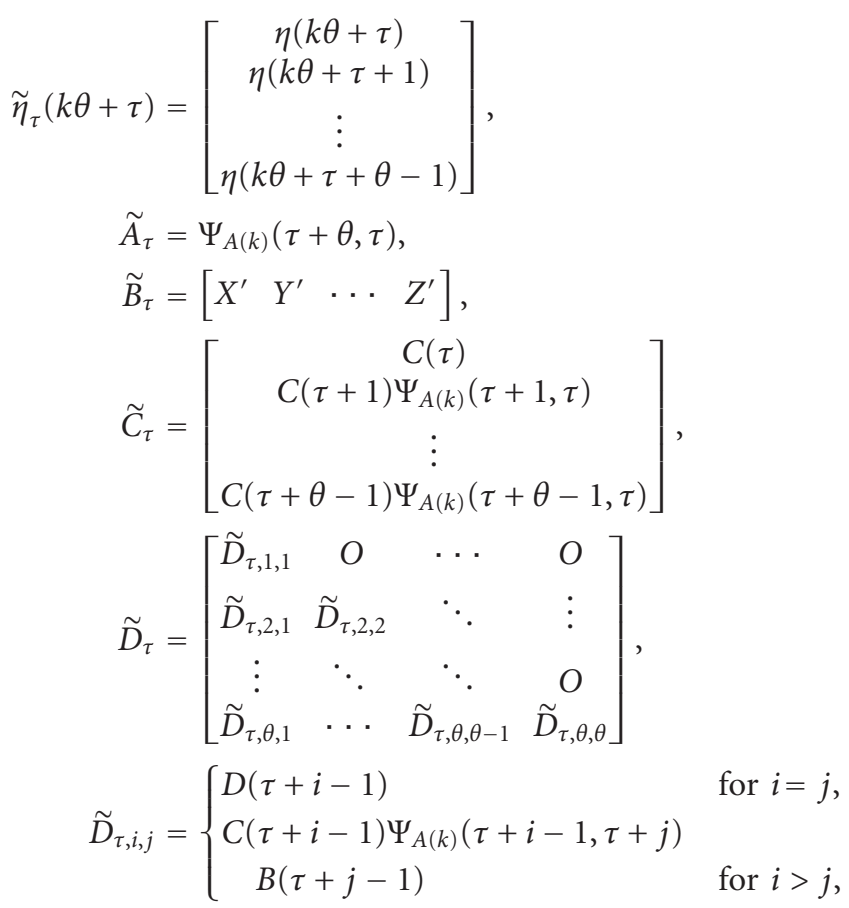


where $\widetilde{B}_{\tau, 1}=\Psi_{A(k)}(\tau+\theta, \tau+1) B(\tau), \widetilde{B}_{\tau, 2}=\Psi_{A(k)}(\tau+\theta, \tau+$ 2) $B(\tau+1), \widetilde{B}_{\tau, \theta}=B(\tau+\theta-1)$, and $\widetilde{E}_{d, \tau}, \widetilde{F}_{d, \tau}$ and $\widetilde{E}_{f, \tau}, \widetilde{F}_{f, \tau}$ are defined in a way similar to $\widetilde{C}_{\tau}, \widetilde{D}_{\tau}$.

\subsubsection{Observer-based FD system design and implementation}

Assume that $(A(k), C(k))$ is detectable. Then $\left(\tilde{A}_{\tau}, \widetilde{C}_{\tau}\right)$ is detectable and an observer-based LTI residual generator can be designed based on lifted reformulation (74) as

$$
\begin{aligned}
\hat{\tilde{x}}_{\tau}((k+1) \theta+\tau)= & \widetilde{A}_{\tau} \hat{\tilde{x}}_{\tau}(k \theta+\tau)+\widetilde{B}_{\tau} \tilde{u}_{\tau}(k \theta+\tau) \\
& +L_{\tau}\left(\tilde{y}_{\tau}(k \theta+\tau)-\hat{\tilde{y}}_{\tau}(k \theta+\tau)\right), \\
r_{\tau}(k \theta+\tau)= & W_{\tau}\left(\tilde{y}_{\tau}(k \theta+\tau)-\hat{\tilde{y}}_{\tau}(k \theta+\tau)\right), \\
\hat{\tilde{y}}_{\tau}(k)= & \widetilde{C}_{\tau} \hat{\tilde{x}}_{\tau}(k \theta+\tau)+\tilde{D}_{\tau} \tilde{u}_{\tau}(k \theta+\tau),
\end{aligned}
$$

where $L_{\tau}$ and $W_{\tau}$ are constant matrices and can be designed with FD approaches for the discrete LTI systems introduced in the last section to realize full decoupling or optimal FD. Observer (76) reconstructs the outputs over one period $y(k \theta+\tau), \ldots, y(k \theta+\tau+\theta-1)$ based on the estimation of state vector $x(k \theta+\tau)$. Both the state vector of observer (76) and the residual signal are updated every $\theta$ time instants.

In fault detection, the detection delay should be as small as possible. Therefore, it is advantageous if a residual signal can be obtained at each time instant. To this aim,

(i) a bank of LTI residual generators (76) can be used, each of which is designed for $\tau=0,1, \ldots, \theta-1$, respectively [43]. This scheme is characterized by a simple design but needs much online computational efforts.

(ii) If the weighting matrix $W_{\tau}$ is designed to satisfy the causality constraint, that is, $W_{\tau}$ is a lower block triangular matrix in the form of

$$
W_{\tau}=\left[\begin{array}{cccc}
W_{\tau, 1,1} & O & \cdots & O \\
W_{\tau, 2,1} & W_{\tau, 2,2} & \ddots & \vdots \\
\vdots & \ddots & \ddots & O \\
W_{\tau, \theta, 1} & \cdots & W_{\tau, \theta, \theta-1} & W_{\tau, \theta, \theta}
\end{array}\right]
$$

then, for a given integer $\tau$, the residual generator (76) can be implemented as

$$
\begin{aligned}
& \hat{\tilde{x}}_{\tau}((k+1) \theta+\tau) \\
& =\tilde{A}_{\tau} \hat{\tilde{x}}_{\tau}(k \theta+\tau)+\widetilde{B}_{\tau} \tilde{u}_{\tau}(k \theta+\tau) \\
& \quad+L_{\tau}\left(\left[\begin{array}{c}
y(k \theta+\tau) \\
\vdots \\
y(k \theta+\tau+\theta-1)
\end{array}\right]-\left[\begin{array}{c}
\hat{y}(k \theta+\tau) \\
\vdots \\
\hat{y}(k \theta+\tau+\theta-1)
\end{array}\right]\right)
\end{aligned}
$$

$$
\begin{aligned}
& r(k \theta+\tau+j) \\
& =\left[\begin{array}{lll}
W_{\tau, j+1,1} & \cdots & W_{\tau, j+1, j+1}
\end{array}\right] \\
& \times\left(\left[\begin{array}{c}
y(k \theta+\tau) \\
\vdots \\
y(k \theta+\tau+j)
\end{array}\right]-\left[\begin{array}{c}
\hat{y}(k \theta+\tau) \\
\vdots \\
\hat{y}(k \theta+\tau+j)
\end{array}\right]\right), \\
& \hat{y}(k \theta+\tau+j) \\
& =C(\tau+j) \Psi_{A(k)}(\tau+j, \tau) \hat{\tilde{x}}_{\tau}(k \theta+\tau) \\
& +\left[\begin{array}{lll}
\tilde{D}_{\tau, j+1,1} & \cdots & \tilde{D}_{\tau, j+1, j+1}
\end{array}\right]\left[\begin{array}{c}
u(k \theta+\tau) \\
\vdots \\
u(k \theta+\tau+j)
\end{array}\right], \\
& j=0,1, \ldots, \theta-1 \text {. }
\end{aligned}
$$

In this case, the state estimation is still updated at every $\theta$ time instants, but at each time instant $k \theta+\tau+j$, $j=0,1, \ldots, \theta-1$, a residual signal $r(k \theta+\tau+j)$ is calculated from control input and measured outputs available up to the time instant $k \theta+\tau+j$.

\subsubsection{Parity-relation-based FD system design and implementation}

Similarly, a parity-relation-based LTI residual generator can be built as follows:

$$
\begin{aligned}
& r_{\tau}(k \theta+\tau)=V_{\tau, s}\left(\tilde{y}_{\tau, k, s}-\tilde{H}_{u, s} \tilde{u}_{\tau, k, s}\right), \\
& \tilde{y}_{\tau, k, s}=\left[\begin{array}{c}
\tilde{y}_{\tau}((k-s) \theta+\tau) \\
\tilde{y}_{\tau}((k-s+1) \theta+\tau) \\
\vdots \\
\tilde{y}_{\tau}(k \theta+\tau)
\end{array}\right], \\
& \tilde{u}_{\tau, k, s}=\left[\begin{array}{c}
\tilde{u}_{\tau}((k-s) \theta+\tau) \\
\tilde{u}_{\tau}((k-s+1) \theta+\tau) \\
\vdots \\
\tilde{u}_{\tau}(k \theta+\tau)
\end{array}\right] \text {, } \\
& \tilde{H}_{u, s}=\left[\begin{array}{cccc}
\widetilde{D}_{\tau} & O & \cdots & O \\
\widetilde{C}_{\tau} \widetilde{B}_{\tau} & \widetilde{D}_{\tau} & \ddots & \vdots \\
\vdots & \ddots & \ddots & O \\
\widetilde{C}_{\tau} \widetilde{A}_{\tau}^{s-1} \widetilde{B}_{\tau} & \cdots & \widetilde{C}_{\tau} \widetilde{B}_{\tau} & \widetilde{D}_{\tau}
\end{array}\right] \text {, }
\end{aligned}
$$

where $V_{\tau, s}$ is a constant parity matrix,

$$
V_{\tau, s}\left[\begin{array}{c}
\widetilde{C}_{\tau} \\
\widetilde{C}_{\tau} \widetilde{A}_{\tau} \\
\vdots \\
\widetilde{C}_{\tau} \widetilde{A}_{\tau}^{s}
\end{array}\right]=0 .
$$

To get a residual signal at each time instant, we can use a bank of parity-relation-based residual generators, each one 
for $\tau=0,1, \ldots, \theta-1$, respectively. Alternatively, we can also impose a structural constant on parity matrix $V_{\tau, s}$ as

$$
\begin{aligned}
& V_{\tau, s}=\left[\begin{array}{lllll}
V_{\tau, s, 0} & V_{\tau, s, 1} & \cdots & V_{\tau, s, s-1} & V_{\tau, s, s}
\end{array}\right], \\
& V_{\tau, s, s}=\left[\begin{array}{cccc}
\left(V_{\tau, s, s}\right)_{1,1} & O & \cdots & O \\
\left(V_{\tau, s, s}\right)_{2,1} & \left(V_{\tau, s, s}\right)_{2,2} & \ddots & \vdots \\
\vdots & \ddots & \ddots & O \\
\left(V_{\tau, s, s}\right)_{\theta, 1} & \cdots & \left(V_{\tau, s, s}\right)_{\theta, \theta-1} & \left(V_{\tau, s, s}\right)_{\theta, \theta}
\end{array}\right] \text {, }
\end{aligned}
$$

that is, the last block in $V_{\tau, s}$ is a lower triangular matrix, then for a fixed $\tau$, residual generator (79) can be implemented in such a way that only control inputs and measured outputs available up to the time instant $k \theta+\tau+j$ are needed for the calculation of $r(k \theta+\tau+j), j=0,1, \ldots, \theta-1$.

\subsection{FD schemes based on periodic model}

In this subsection, we will show that the parity-space approach and the observer-based FD approach can be directly extended to periodic systems, which do not need the temporary step of lifted LTI reformulation and lead to a simplified design and implementation.

\subsubsection{Periodic parity-space approach}

The extension of the parity-space approach to periodic systems is straightforward, because the parity-space approach can handle each time instant independently [45]. The inputoutput relation of periodic system (72) during the moving window $[k-s, k]$ can be expressed by

$$
y_{k, s}=H_{o, s, k} x(k-s)+H_{u, s, k} u_{k, s}+H_{d, s, k} d_{k, s}+H_{f, s, k} f_{k, s} .
$$

While the vectors $y_{k, s}, u_{k, s}, d_{k, s}$, and $f_{k, s}$ in (82) are built in exactly the same way as in the LTI case according to (6), the matrices $H_{o, s, k}, H_{u, s, k}, H_{d, s, k}$, and $H_{f, s, k}$ in (82) are not constant matrices but the following periodic matrices:

$$
\begin{aligned}
& H_{o, s, k}\left[\begin{array}{c}
C(k-s) \\
C(k-s+1) A(k-s) \\
\vdots \\
C(k) A(k-1) \cdots A(k-s+1) A(k-s)
\end{array}\right], \\
& H_{u, s, k}=\left[\begin{array}{cccc}
D(k-s) & O & \cdots & O \\
C(k-s+1) B(k-s) & D(k-s+1) & \ddots & \vdots \\
\vdots & \ddots & \ddots & O \\
C(k) A(k-1) \cdots & \cdots & & D(k) \\
A(k-s+1) B(k-s) & & &
\end{array}\right] \text {, }
\end{aligned}
$$

$H_{d, s, k}$, and $H_{f, s, k}$ are similar as $H_{u, s, k}$ with $B(j), D(j)$ replaced, respectively, by $E_{d}(j), F_{d}(j)$, and $E_{f}(j), F_{f}(j), j=k-s, \ldots, k$. A periodic residual generator can be built as

$$
\begin{aligned}
& r(k)=V_{k}\left(y_{k, s}-H_{u, s, k} u_{k, s}\right), \\
& \stackrel{(\mathrm{a})}{=} V_{k}\left(H_{d, s, k} d_{k, s}+H_{f, s, k} f_{k, s}\right),
\end{aligned}
$$

where $V_{k}$ is a $\theta$-periodic parity matrix (or vector) that satisfies $V_{k} H_{o, s, k}=0$, equation (85) represents the residual dynamics.

If the rank condition

$$
\operatorname{rank}\left[\begin{array}{lll}
H_{o, s, k} & H_{d, s, k} & H_{f, s, k}
\end{array}\right]>\operatorname{rank}\left[\begin{array}{ll}
H_{o, s, k} & H_{d, s, k}
\end{array}\right]
$$

holds for any $k$, then a full decoupling can be achieved by solving

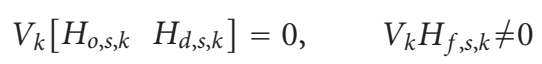

for $V_{k}$ over one period at $k, k+1, \ldots, k+\theta-1$. The residual evaluation consists in detecting the deviation of residual $r(k)$ from 0 . Especially, if

$$
\begin{gathered}
\operatorname{rank}\left[\begin{array}{lllll}
H_{o, s, 0} \cdots H_{o, s, \theta-1} & H_{d, s, 0} \cdots & H_{d, s, \theta-1} & H_{f, s, 0} \cdots H_{f, s, \theta-1}
\end{array}\right] \\
>\operatorname{rank}\left[\begin{array}{llllll}
H_{o, s, 0} & \cdots & H_{o, s, \theta-1} & H_{d, s, 0} & \cdots & H_{d, s, \theta-1}
\end{array}\right]
\end{gathered}
$$

then the full decoupling can be achieved by a constant parity matrix (or vector) $V_{k}$. However, condition (88) is rather restrictive in practice.

In case that a full decoupling is not achievable, optimization problems similar to (15)-(17) are formulated as

$$
\begin{aligned}
\max _{V_{k}, V_{k} H_{o, s, k}=0} J_{\mathrm{LTP}, \mathrm{PS}, \infty / \infty}\left(V_{k}\right) & =\max _{V_{k}, V_{k} H_{o, s, k}=0} \frac{\bar{\sigma}^{2}\left(V_{k} H_{f, s, k}\right)}{\bar{\sigma}^{2}\left(V_{k} H_{d, s, k}\right)}, \\
\max _{V_{k}, V_{k} H_{o, s, k}=0} J_{\mathrm{LTP}, \mathrm{PS},-/ \infty}\left(V_{k}\right) & =\max _{V_{k}, V_{k} H_{o, s, k}=0} \frac{\sigma^{2}\left(V_{k} H_{f, s, k}\right)}{\bar{\sigma}^{2}\left(V_{k} H_{d, s, k}\right)}, \\
\max _{V_{k}, V_{k} H_{o, s, k}=0} J_{\mathrm{LTP}, \mathrm{PS}, i / \infty}\left(V_{k}\right) & =\max _{V_{k}, V_{k} H_{o, s, k}=0} \frac{\sigma_{i}^{2}\left(V_{k} H_{f, s, k}\right)}{\bar{\sigma}^{2}\left(V_{k} H_{d, s, k}\right)},
\end{aligned}
$$

which are solved over one period to get the optimal periodic parity matrix $V_{k}$. Because the parity-space approach handles each time instant independently and there is no stability problem, the solutions of problems (87)-(91) at each time instant are independent of each other and can be obtained following the procedures introduced in Section 2.2. The threshold Jth can be calculated by (23) or (26) according to the requirement on FAR, while the residual evaluation function is selected as the amplitude (22) of the residual signal.

If the order $s$ of the parity relation (82) is an integer multiple of the period $\theta$, then the periodic parity-space approach is equivalent with a bank of residual generators (79). In comparison, the periodic parity space approach provides more flexibility. The order of the parity relation $s$ needs not to be related to the period $\theta$. Moreover, $s$ may take different values at different time instants. In this case, the threshold for the residual evaluation may need to be chosen differently at different time. 


\subsubsection{Periodic observer-based approach}

Assume that $(A(k), C(k))$ is detectable. A periodic observerbased residual generator can be constructed as

$$
\begin{aligned}
\hat{x}(k+1) & =A(k) \hat{x}(k)+B(k) u(k)+L(k)(y(k)-\hat{y}(k)), \\
r(k) & =W(k)(y(k)-\hat{y}(k)), \\
\hat{y}(k) & =C(k) \hat{x}(k)+D(k) u(k),
\end{aligned}
$$

where $L(k)$ and $W(k)$ are $\theta$-periodic observer gain matrix and weighting matrix, respectively. The residual dynamics is governed by

$$
\begin{aligned}
e(k+1)= & (A(k)-L(k) C(k)) e(k)+\left(E_{d}(k)-L(k) F_{d}(k)\right) d(k) \\
& +\left(E_{f}(k)-L(k) F_{f}(k)\right) f(k), \\
r(k)= & W(k)\left(C(k) e(k)+F_{d}(k) d(k)+F_{f}(k) f(k)\right),
\end{aligned}
$$

where $e(k)=x(k)-\hat{x}(k)$.

To enhance the robustness of the FD system to the unknown disturbances without loss of the sensitivity to the faults, the optimal design problem is formulated as

$$
\begin{aligned}
& \sup _{L(k), W(k)} J_{\mathrm{LTP}, \mathrm{OBS}, \infty / \infty}(L(k), W(k)) \\
& =\sup _{L(k), W(k)} \frac{\sup _{d=0, f \in l_{2}-\{0\}}\left(\|r\|_{2} /\|f\|_{2}\right)}{\sup _{f=0, d \in l_{2}-\{0\}}\left(\|r\|_{2} /\|d\|_{2}\right)}, \\
& \sup _{L(k), W(k)} J_{\mathrm{LTP}, \mathrm{OBS},-/ \infty}(L(k), W(k)) \\
& =\sup _{L(k), W(k)} \frac{\inf _{d=0, f \in l_{2}-\{0\}}\left(\|r\|_{2} /\|f\|_{2}\right)}{\sup _{f=0, d \in l_{2}-\{0\}}\left(\|r\|_{2} /\|d\|_{2}\right)} .
\end{aligned}
$$

The solutions of optimization problems (94)-(95) are derived by solving an equivalent optimization problem for the cyclically lifted LTI systems first and then recover the periodic matrices $L(k)$ and $W(k)[24]$.

\section{Solution to optimization problems (94)-(95)}

Assume that $\left(A(k), E_{d}(k), C(k), F_{d}(k)\right)$ is regular. Then

$$
L(k)=-L_{d}^{T}(k), \quad W(k)=W_{d}(k)
$$

solve optimization problems (94)-(95) simultaneously, where $W_{d}(k)$ is the left inverse of a full column rank matrix $H_{d}(k)$ satisfying $H_{d}(k) H_{d}^{T}(k)=C(k) X_{d}(k) C^{T}(k)+$ $F_{d}(k) F_{d}^{T}(k)$, and $\left(X_{d}(k), L_{d}(k)\right)$ is the stabilizing solution to the difference periodic Riccati system (DPRS)

$$
\left[\begin{array}{ll}
\Omega_{d, 11} & \Omega_{d, 12} \\
\Omega_{d, 12}^{T} & \Omega_{d, 22}
\end{array}\right] \times\left[\begin{array}{c}
I \\
L_{d}(k)
\end{array}\right]=0,
$$

where $\Omega_{d, 11}=A(k) X_{d}(k) A^{T}(k)-X_{d}(k+1)+E_{d}(k) E_{d}^{T}(k)$, $\Omega_{d, 12}=A(k) X_{d}(k) C^{T}(k)+E_{d}(k) F_{d}^{T}(k)$, and $\Omega_{d, 22}=$ $C(k) X_{d}(k) C^{T}(k)+F_{d}(k) F_{d}^{T}(k)$. An alternative solution to problem (95), if $\left(A(k), E_{f}(k), C(k), F_{f}(k)\right)$ is regular, is given by

$$
L(k)=-L_{f}^{T}(k), \quad W(k)=W_{f}(k),
$$

where $W_{f}(k)$ is the left inverse of a full column rank matrix $H_{f}(k)$ satisfying $H_{f}(k) H_{f}^{T}(k)=C(k) X_{f}(k) C^{T}(k)+$ $F_{f}(k) F_{f}^{T}(k)$, and $\left(X_{f}(k), L_{f}(k)\right)$ is the stabilizing solution to the DPRS

$$
\left[\begin{array}{ll}
\Omega_{f, 11} & \Omega_{f, 12} \\
\Omega_{f, 12}^{T} & \Omega_{f, 22}
\end{array}\right] \times\left[\begin{array}{c}
I \\
L_{f}(k)
\end{array}\right]=0
$$

where $\Omega_{f, 11}=A(k) X_{f}(k) A^{T}(k)-X_{f}(k+1)+E_{f}(k) E_{f}^{T}(k)$, $\Omega_{f, 12}=A(k) X_{f}(k) C^{T}(k)+E_{f}(k) F_{f}^{T}(k)$, and $\Omega_{f, 22}=$ $C(k) X_{f}(k) C^{T}(k)+F_{f}(k) F_{f}^{T}(k)$. It is interesting to note the following connections between different approaches.

(i) Periodic observer-based residual generator (92) can be rewritten into the form of lifted reformulation-based LTI observer (76) with

$$
\begin{aligned}
L_{\tau}= & {\left[\begin{array}{llll}
L_{\tau, 1} & L_{\tau, 2} & \cdots & L_{\tau, \theta}
\end{array}\right], } \\
L_{\tau, i}= & \Psi_{A(k)-L(k) C(k)}(\tau+\theta, \tau+i) L(\tau+i-1), \\
W_{\tau}= & {\left[\begin{array}{cccc}
W_{\tau, 1,1} & O & \cdots & O \\
W_{\tau, 2,1} & W_{\tau, 2,2} & \ddots & \vdots \\
\vdots & \ddots & \ddots & O \\
W_{\tau, \theta, 1} & \cdots & W_{\tau, \theta, \theta-1} & W_{\tau, \theta, \theta}
\end{array}\right], } \\
W_{\tau, i, j}= & W_{(\tau+i-1),} \text { if } i=j, \\
W_{\tau, i, j}= & -W(\tau+i-1) C(\tau+i-1) \\
& \times \Psi_{A(k)-L(k) C(k)}(\tau+i-1, \tau+j) L(\tau+j-1) \\
& \text { if } i>j, i=1,2, \ldots, \theta, j=1,2, \ldots, \theta .
\end{aligned}
$$

Recalling the discussion in Section 3.1.1, the physical meaning is that the periodic observer-based residual generator naturally satisfies the causality condition. It is further proven that, if the parameters $(L(k), W(k))$ of the periodic observer-based residual generator (92) solve optimization problems (94) or (95), then the parameters $\left(L_{\tau}, W_{\tau}\right)$ of the LTI observer (76) got by (100) will solve optimization problems in the form of (60)(61).

(ii) Similar as in LTI systems, the periodic parity-space approach and periodic observer-based approach are closely related. Assume that the periodic vector

$$
v_{k}=\left[\begin{array}{llll}
v_{k, 0} & v_{k, 1} & \cdots & v_{k, s}
\end{array}\right]
$$

satisfies $v_{k} H_{o, s, k}=0$. Then a periodic functional observer-based residual generator in the form of

$$
\begin{gathered}
z(k+1)=G(k) z(k)+H(k) u(k)+L(k) y(k), \\
r(k)=w(k) z(k)+q(k) u(k)+p(k) y(k)
\end{gathered}
$$


with $z \in \mathcal{R}^{s}, r \in \mathcal{R}$, can be readily obtained as [45]

$$
\begin{aligned}
& G(k)=\left[\begin{array}{ccccc}
0 & 0 & \cdots & 0 & g_{k, 1} \\
1 & 0 & \ddots & \vdots & g_{k, 2} \\
\vdots & \ddots & \ddots & 0 & \vdots \\
0 & \cdots & 1 & 0 & g_{k, s-1} \\
0 & \cdots & 0 & 1 & g_{k, s}
\end{array}\right] \text {, } \\
& L(k)=-\left[\begin{array}{c}
v_{k+s, 0} \\
v_{k+s-1,1} \\
\vdots \\
v_{k+1, s-1}
\end{array}\right]-\left[\begin{array}{c}
g_{k, 1} \\
g_{k, 2} \\
\vdots \\
g_{k, s}
\end{array}\right] v_{k, s} \\
& w(k)=\left[\begin{array}{lllll}
0 & 0 & \cdots & 0 & -1
\end{array}\right], \\
& p(k)=v_{k, s}, \\
& H(k)=T(k+1) B(k)-L(k) D(k), \\
& q(k)=-p(k) D(k),
\end{aligned}
$$

where periodic scalars $g_{k, 1}, \ldots, g_{k, s}$ appearing in matrices $G(k), L(k)$ are free parameters and should be selected to guarantee the stability of $G(k)$. Moreover, if $v_{k}$ realizes a full decoupling from the unknown disturbances, that is, $v_{k}\left[\begin{array}{ll}H_{o, s, k} & H_{d, s, k}\end{array}\right]=0$, then the functional observer-based residual generator (102) also achieves a full decoupling, that is, $r(k)=0$, $\forall u(k), d(k)$. This provides an approach to design full decoupling observer-based residual generator.

(iii) We would like to point out that, for the LTI system (1), a residual generator with periodic gain matrix $L(k)$ and periodic weighting matrix $W(k)$ will not improve the FD performance under performance index (94).

\section{FD OF SAMPLED-DATA SYSTEMS}

The study on FD problems of sampled-data (SD) systems has been motivated by the digital implementation of controllers and monitoring systems. Figure 1 sketches a typical application of an FD system in a process control system. The process under consideration is a continuous-time process. Both the controller and the FD system are discrete-time systems which are implemented on a computer system or on an embedded microprocessor. The sensor output signals are discretized by the A/D converters and then fed to the controller as well as to the FD system. The D/A converters convert the discrete-time control input signals into continuous-time signals. Since both continuous-time and discrete-time signals exist in the system, the system design should be indeed considered from the viewpoint of an SD system [46, 47]. The intersample behavior is the main factor that should be considered in developing FD algorithms for the SD systems. In practice, it happens often that the $\mathrm{A} / \mathrm{D}$ and $\mathrm{D} / \mathrm{A}$ converters are working at different sampling rates [48-53]. In this section, we will review the FD methods for the SD systems with various sampling mechanisms.

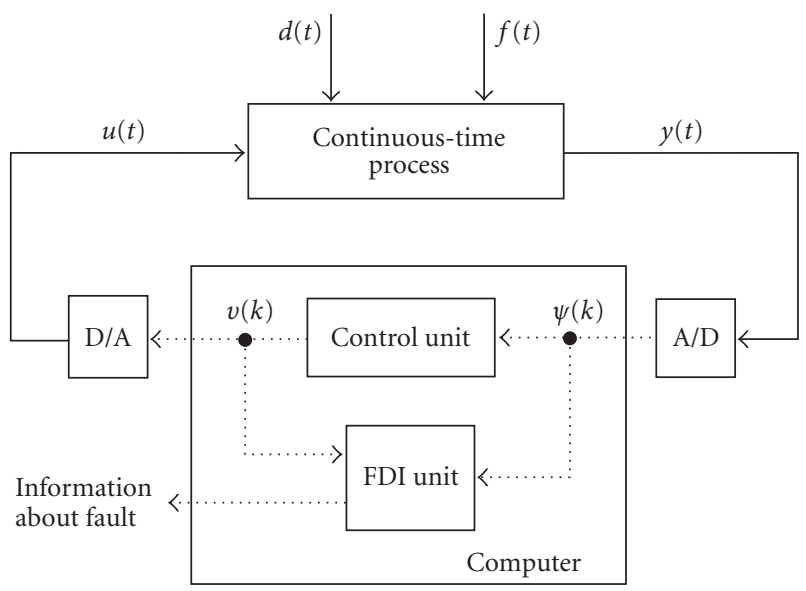

FIGURE 1: Schematic description of the application of an FDI system in a process control system.

\subsection{System description}

Assume that, in the SD systems, the process is a continuous LTI process represented by

$$
\begin{aligned}
& \dot{x}(t)=A_{c} x(t)+B_{c} u(t)+E_{d c} d(t)+E_{f c} f(t), \\
& y(t)=C x(t),
\end{aligned}
$$

where $A_{c}, B_{c}, E_{d c}, E_{f c}$, and $C$ are known constant matrices of appropriate dimensions. In single-rate sampled-data (SSD) systems, the A/D converter and the D/A converter are, respectively, described by

$$
\begin{gathered}
\psi(k)=y(k h), \\
u(t)=v(k), \quad k h \leqslant t<(k+1) h,
\end{gathered}
$$

where $h$ is the sampling period, $\psi \in \mathcal{R}^{m}$ is the sampled process output signal, $v \in \mathcal{R}^{p}$ is the discrete-time control input sequence delivered by the controller program. In $\mathrm{mul}$ tirate sampled-data (MSD) systems, the A/D converters and the D/A converters may work with different sampling rates and thus modeled, respectively, by

$$
\begin{gathered}
\psi_{l}\left(k^{l}\right)=y_{l}\left(k^{l} T_{y, l}\right), \quad l=1,2, \ldots, m ; k^{l}=0,1,2, \ldots \\
u_{j}(t)=v_{j}\left(k^{j}\right), \quad k^{j} T_{u, j} \leq t<\left(k^{j}+1\right) T_{u, j}, \\
j=1,2, \ldots, p ; k^{j}=0,1,2, \ldots
\end{gathered}
$$

where $T_{y, l}$ and $T_{u, j}$ denote, respectively, the sampling periods of the A/D converter in the $l$ th output channel and the $\mathrm{D} / \mathrm{A}$ converter in the $j$ th input channel. A more general class of systems are nonuniformly sampled-data (NSD) systems, where the sampling instants may be multirate, asynchronous, and nonequidistantly distributed, that is,

$$
\begin{aligned}
\psi_{l}\left(k^{l}\right) & =y_{l}\left(t_{y, k^{l}}\right), \quad l=1,2, \ldots, m ; k^{l}=0,1,2, \ldots, \\
u_{j}(t) & =v_{j}\left(k^{j}\right), \quad t_{u, k^{j}} \leq t<t_{u, k^{j}+1}, \\
j & =1,2, \ldots, p ; k^{j}=0,1,2, \ldots,
\end{aligned}
$$


where $t_{y, k^{l}}$ represent the sampling instants in the $l$ th output channel and $t_{u, k^{j}}$ the time instants at which the $j$ th control input is updated. It is worth mentioning that a special kind of NSD systems, where the sampling instants are nonequidistant spaced but periodic, has been studied in the literature rather intensively [54-57].

\subsection{FD of SSD systems}

Conventionally, an FD system can be designed for the SSD system by indirect approaches, that is,

(i) analog design and SD implementation, or

(ii) discrete-time design based on the discretization of the process model.

Motivated by the development of sampled-data control [46, 47], in the last years the FD problem of the SSD systems have been studied from the viewpoint of direct design to take into account the intersample behavior and eliminate the approximation made during the design [26, 58-60].

The dynamics of the SSD system at the sampling instants can be described by

$$
\begin{aligned}
x(k+1) & =A x(k)+B v(k)+\bar{d}(k)+\bar{f}(k), \\
\psi(k) & =C x(k),
\end{aligned}
$$

where

$$
\begin{aligned}
A & =e^{A_{c} h}, \quad B=\int_{0}^{h} e^{A_{c} t} B_{c} d t, \\
\bar{d}(k) & =\int_{0}^{h} e^{A_{c}(h-\tau)} E_{d c} d(k h+\tau) d \tau, \\
\bar{f}(k) & =\int_{0}^{h} e^{A_{c}(h-\tau)} E_{f c} f(k h+\tau) d \tau .
\end{aligned}
$$

It is worth noticing that in SD systems there is a significant difference between $u(t)$ and $d(t), f(t)$. Due to the D/A converter (106), $u(t)$ is a piecewise constant signal. The influence of $u(t)$ on $y(t)$ is exactly known from the information of $v(k)$ and can thus be completely compensated in residual generation. In comparison, $d(t)$ and $f(t)$ are unknown signals. Hence, the key is to study the influence of continuoustime signals $d(t)$ and $f(t)$ on the discrete-time sampled output signals $\psi(k)$ and residual signals $r(k)$.

\subsubsection{Parity-relation-based FD scheme for SSD systems}

A parity-relation-based residual generator

$$
r(k)=V_{s}\left(\psi_{k, s}-H_{u, s} v_{k, s}\right)
$$

can be used for residual generation, where $V_{s} H_{o, s}=0$, $\psi_{k, s}, v_{k, s}, H_{o, s}$, and $H_{u, s}$ are constructed according to (6). To describe the intersample behavior, for a continuous-time sig- nal $\delta(t)$ with $\delta$ standing for $d$ and $f$, an operator is defined as follows:

$$
\begin{aligned}
\Psi^{\delta} \delta_{k, s}(t) & =\left[\begin{array}{c}
\bar{\delta}(k-s) \\
\bar{\delta}(k-s+1) \\
\vdots \\
\bar{\delta}(k)
\end{array}\right] \\
= & {\left[\begin{array}{c}
\int_{0}^{h} e^{A_{c}(h-\tau)} E_{\delta c} \delta((k-s) h+\tau) d \tau \\
\int_{0}^{h} e^{A_{c}(h-\tau)} E_{\delta c} \delta((k-s+1) h+\tau) d \tau \\
\vdots \\
\int_{0}^{h} e^{A_{c}(h-\tau)} E_{\delta c} \delta(k h+\tau) d \tau
\end{array}\right] . }
\end{aligned}
$$

The residual dynamics can be expressed with the help of operators as

$$
\begin{aligned}
r(k) & =V_{s} H\left(\Psi^{d} d_{k, s}(t)+\Psi^{f} f_{k, s}(t)\right), \\
H & =\left[\begin{array}{cccc}
O & O & \cdots & O \\
C & O & \ddots & \vdots \\
\vdots & \ddots & \ddots & O \\
C A^{s-1} & \cdots & C & O
\end{array}\right] .
\end{aligned}
$$

The influence of the continuous-time signal $\delta(t)$ over the time interval $[(k-s) h,(k+1) h)$ on the discrete-time signal $\Psi^{\delta} \delta_{k, s}(t)$ is measured by

$$
\begin{aligned}
& \bar{\sigma}\left(V_{s} H \Psi^{\delta}\left(\Psi^{\delta}\right)^{*} H^{T} V_{s}^{T}\right)=\sup _{\delta \in \mathcal{L}_{2,[(k-s) h,(k+1) h)}} \frac{r^{T}(k) r(k)}{\|\delta\|_{2,[(k-s) h,(k+1) h)}^{2}}, \\
& \underline{\sigma}\left(V_{s} H \Psi^{\delta}\left(\Psi^{\delta}\right)^{*} H^{T} V_{s}^{T}\right)=\inf _{\delta \in \mathscr{L}_{2,[(k-s) h,(k+1) h)}} \frac{r^{T}(k) r(k)}{\|\delta\|_{2,[(k-s) h,(k+1) h)}^{2}},
\end{aligned}
$$

where $\left(\Psi^{\delta}\right)^{*}$ denotes the adjoint of the operator $\Psi^{\delta}$ which uniquely satisfies

$$
\left\langle\Psi^{\delta} \delta_{k, s}(t), \beta_{s}(k)\right\rangle=\left\langle\delta_{k, s}(t),\left(\Psi^{\delta}\right)^{*} \beta_{s}(k)\right\rangle
$$

for any vector $\beta_{s}(k)$ of compatible dimensions. The optimization problems are thus formulated as

$$
\begin{aligned}
& \max _{V_{s}, V_{s} H_{o, s}=0} J_{\text {SSD }, \mathrm{PS}, \infty / \infty}\left(V_{s}\right)=\max _{V_{s}, V_{s} H_{o, s}=0} \frac{\bar{\sigma}\left(V_{s} H \Psi^{f}\left(\Psi^{f}\right)^{*} H^{T} V_{s}^{T}\right)}{\bar{\sigma}\left(V_{s} H \Psi^{d}\left(\Psi^{d}\right)^{*} H^{T} V_{s}^{T}\right)}, \\
& \max _{V_{s}, V_{s} H_{o, s}=0} J_{\text {SSD }, \mathrm{PS},-/ \infty}\left(V_{s}\right)=\max _{V_{s}, V_{s} H_{o, s}=0} \frac{\underline{\sigma}\left(V_{s} H \Psi^{f}\left(\Psi^{f}\right)^{*} H^{T} V_{s}^{T}\right)}{\bar{\sigma}\left(V_{s} H \Psi^{d}\left(\Psi^{d}\right)^{*} H^{T} V_{s}^{T}\right)}, \\
& \max _{V_{s}, V_{s} H_{o, s}=0} J_{\text {SSD,PS }, i / \infty}\left(V_{s}\right)=\max _{V_{s}, V_{s} H_{o, s}=0} \frac{\sigma_{i}\left(V_{s} H \Psi^{f}\left(\Psi^{f}\right)^{*} H^{T} V_{s}^{T}\right)}{\bar{\sigma}\left(V_{s} H \Psi^{d}\left(\Psi^{d}\right)^{*} H^{T} V_{s}^{T}\right)} .
\end{aligned}
$$

An analytical expression can be obtained for $\Psi^{\delta}\left(\Psi^{\delta}\right)^{*}$ as

$$
\begin{aligned}
\Psi^{\delta}\left(\Psi^{\delta}\right)^{*} & =\operatorname{diag}\left\{\bar{E}_{\delta} \bar{E}_{\delta}^{T}, \bar{E}_{\delta} \bar{E}_{\delta}^{T}, \ldots, \bar{E}_{\delta} \bar{E}_{\delta}^{T}\right\}, \\
\bar{E}_{\delta} \bar{E}_{\delta}^{T} & =\int_{0}^{h} e^{A_{c} \tau} E_{\delta c} E_{\delta c}^{T} e^{A_{c}^{T} \tau} d \tau .
\end{aligned}
$$


As a result, optimization problems (117) are transformed into some equivalent optimization problems:

$$
\begin{array}{r}
\max _{V_{s}, V_{s} H_{o, s}=0} J_{\text {SSD }, \mathrm{PS}, \infty / \infty}\left(V_{s}\right)=\max _{V_{s}, V_{s} H_{o, s}=0} \frac{\bar{\sigma}^{2}\left(V_{s} \bar{H}_{f, s}\right)}{\bar{\sigma}^{2}\left(V_{s} \bar{H}_{d, s}\right)}, \\
\max _{V_{s}, V_{s} H_{o, s}=0} J_{\text {SSD }, \mathrm{PS},-/ \infty}\left(V_{s}\right)=\max _{V_{s}, V_{s} H_{o, s}=0} \frac{\sigma^{2}\left(V_{s} \bar{H}_{f, s}\right)}{\bar{\sigma}^{2}\left(V_{s} \bar{H}_{d, s}\right)}, \\
\max _{V_{s}, V_{s} H_{o, s}=0} J_{\mathrm{SSD}, \mathrm{PS}, i / \infty}\left(V_{s}\right)=\max _{V_{s}, V_{s} H_{o, s}=0} \frac{\sigma_{i}^{2}\left(V_{s} \bar{H}_{f, s}\right)}{\bar{\sigma}^{2}\left(V_{s} \bar{H}_{d, s}\right)},
\end{array}
$$

where $\bar{H}_{f, s}$ and $\bar{H}_{d, s}$ are built based on $\left(A, \bar{E}_{f}, C, O\right)$ and $\left(A, \bar{E}_{d}, C, O\right)$ in a way similar to $H_{u, s}$ in (6). The equivalent optimization problems (119) are of the standard form and can be solved as introduced in Section 2.2.

\subsubsection{Observer-based FD scheme for SSD systems}

An observer-based residual generator is constructed as

$$
\begin{aligned}
\hat{x}(k+1) & =A \hat{x}(k)+B v(k)+L(\psi(k)-\widehat{\psi}(k)), \\
r(k) & =W(\psi(k)-\hat{\psi}(k)) \quad \hat{\psi}(k)=C \hat{x}(k) .
\end{aligned}
$$

To describe the influence of continuous-time signals $d(t)$ and $f(t)$ on the discrete-time residual signal $r(k)$ in the frequency domain, operator $\Gamma_{\delta}(\delta$ standing for $d$ or $f)$ is introduced as

$$
\begin{gathered}
\Gamma_{\delta} \delta(j \omega)=\frac{1}{h} \sum_{k=-\infty}^{\infty} G_{\delta}\left(j \omega+j k \omega_{s}\right) \delta\left(j \omega+j k \omega_{s}\right), \\
G_{\delta}(s)=C\left(s I-A_{c}\right)^{-1} E_{\delta c}, \quad \omega_{s}=\frac{2 \pi}{h} .
\end{gathered}
$$

Based on it, the residual dynamics can be expressed as

$$
\begin{aligned}
r\left(e^{j \omega h}\right) & =W \widehat{M}_{u}\left(e^{j \omega h}\right)\left(\Gamma_{d} d(j \omega)+\Gamma_{f} f(j \omega)\right), \\
\widehat{M}_{u}\left(e^{j \omega h}\right) & =I-C\left(e^{j \omega h} I-A+L C\right)^{-1} L .
\end{aligned}
$$

Let $\bar{E}_{\delta}$ be given by (118). Then

$$
\Gamma_{\delta} \Gamma_{\delta}^{*}=C\left(e^{j \omega h} I-A\right)^{-1} \bar{E}_{\delta} \bar{E}_{\delta}^{T}\left(e^{-j \omega h} I-A^{T}\right)^{-1} C^{T} .
$$

Based on it, the $\mathrm{H}_{2} / \mathrm{H}_{2}$ optimal design problem is solved [59]. Further, it was shown that the $H_{\infty} / H_{\infty}, H_{-} / H_{\infty}$ and $H_{i} / H_{\infty}$ design problems of the SSD system are equivalent to that of a discrete LTI system and can be obtained by solving equivalent optimization problems $[26,60]$ :

$$
\begin{aligned}
\sup _{L, W} J_{\text {SSD }, \mathrm{OBS}, \infty / \infty}(L, W) & =\sup _{L, W} \frac{\sup _{d=0, f \in \mathcal{L}_{2}-\{0\}}\left(\|r\|_{2} /\|f\|_{2}\right)}{\sup _{f=0, d \in \mathcal{L}_{2}-\{0\}}\left(\|r\|_{2} /\|d\|_{2}\right)} \\
& =\sup _{L, W} \frac{\left\|\bar{G}_{r f}(z)\right\|_{\infty}}{\left\|\bar{G}_{r d}(z)\right\|_{\infty}}, \\
\sup _{L, W} J_{\text {SSD }, \mathrm{OBS},-/ \infty}(L, W) & =\sup _{L, W} \frac{\inf _{d=0, f \in \mathscr{L}_{2}-\{0\}}\left(\|r\|_{2} /\|f\|_{2}\right)}{\sup _{f=0, d \in \mathcal{L}_{2}-\{0\}}\left(\|r\|_{2} /\|d\|_{2}\right)} \\
& =\sup _{L, W} \frac{\left\|\bar{G}_{r f}(z)\right\|_{-}}{\left\|\bar{G}_{r d}(z)\right\|_{\infty}}, \\
\sup _{L, W} J_{\mathrm{SSD}, \mathrm{OBS}, i / \infty}(L, W) & =\sup _{L, W} \frac{\sigma_{i}\left(\bar{G}_{r f}\left(e^{j \omega h}\right)\right)}{\left\|\bar{G}_{r d}(z)\right\|_{\infty}} \\
\bar{G}_{r \delta}(z) & =W C(z I-A+L C)^{-1} \bar{E}_{\delta}, \quad \delta=d, f .
\end{aligned}
$$

\subsubsection{Influence of sampling on full decoupling}

No matter which residual generation approach is adopted, due to the sampling effect, the full decoupling becomes more difficult in SD systems than in the original continuous-time systems, because after sampling the dimension of the influence space of the unknown disturbances becomes

$$
\begin{aligned}
\operatorname{rank} \bar{E}_{d} & =\operatorname{rank} \bar{E}_{d} \bar{E}_{d}^{T}=\operatorname{rank} \int_{0}^{h} e^{A_{c} \tau} E_{d c} E_{d c}^{T} e^{A_{c}^{T} \tau} d \tau \\
& \geq \operatorname{rank} E_{d c}
\end{aligned}
$$

that is, the equivalent number of the unknown disturbances may increase [58].

\subsection{FD of MSD systems}

Under some assumptions, the FD problem of MSD systems has been considered in [61-64]. The MSD system is in nature a periodic system. The system period, denoted by $T$, is the least common multiple of the sampling periods [52]. The maximal common multiplier of the sampling periods is often called base period. From the FD viewpoint, in MSD systems only those time instants with sampled outputs are of interest. In [65], the basic idea of the proposed FD approach is to get the input-output relations of the MSD at the base periods at first and then downsample them according to different sampling periods to get the parity relation of the MSD system. In [66], a so-called fast rate residual generator is proposed, which generates a residual signal as soon as a new measurement is available. The basic idea is to reformulate the MSD system as a system with periodic output equations. The problem of fast rate residual generation is further pursued by $[67,68]$, where the basic ideas are, respectively, to compute the parity matrix $V_{s}$ and the postfilter $R(z)$ for lifted system model. To satisfy the causality constraint, the freedoms in matrix $\bar{P}_{s}$ and $R(z)$ are used. Most recently, a unified and simplified approach to the FD of the MSD systems is proposed by [69], which considers the special problem formulation of fault detection and shows clearly the difference between control problem and FD problem. The basic idea of [69] is to remodel the MSD system as a nonuniformly sampled-data system and then use periodic or time varying system theory to design the FD system.

Denote with $\left\{t_{k}\right\}$ the sequence of time instants at which one or more sampled outputs are available, $t_{0}<t_{1}<\cdots<$ $t_{k}<t_{k+1}<\cdots$. Let $\bar{\psi}(k)$ represent the vector of sampled output signals at time instant $t_{k}$. The dimension of $\bar{\psi}(k)$ is time-varying and upper bounded by $m$. Let $x(k)=x\left(t_{k}\right)$. For the purpose of FD, the MSD system described by (104), (107), and (108) can be equivalently remodeled as

$$
\begin{aligned}
x(k+1) & =A(k) x(k)+\bar{u}(k)+\bar{d}(k)+\bar{f}(k), \\
\bar{\psi}(k) & =C(k) x(k),
\end{aligned}
$$


where

$$
\begin{aligned}
A(k) & =e^{A_{c}\left(t_{k+1}-t_{k}\right)}, \\
\bar{u}(k) & =\int_{t_{k}}^{t_{k+1}} e^{A_{c}\left(t_{k+1}-t\right)} B_{c} u(t) d t, \\
\bar{d}(k) & =\int_{t_{k}}^{t_{k+1}} e^{A_{c}\left(t_{k+1}-t\right)} E_{d c} d(t) d t, \\
\bar{f}(k) & =\int_{t_{k}}^{t_{k+1}} e^{A_{c}\left(t_{k+1}-t\right)} E_{f c} f(t) d t .
\end{aligned}
$$

The new description is different from other descriptions available in the literature [66-68] and considers the transition of system dynamics only at the time instants with sampled outputs. The terms $\bar{d}(k)$ and $\bar{f}(k)$ characterize, respectively, the influence of the disturbances and the faults on the sampled outputs. As $v_{1}, \ldots, v_{p}$ are available and the models of the D/A converters (108) are known, $\bar{u}(k)$ and thus the influence of the control input vector on the sampled outputs can be completely reconstructed and easily compensated. The matrices $A(k)$ and $C(k)$ are time varying matrices, as $t_{k+1}-t_{k}$ is time-varying with respect to time $k$. In the MSD system, due to the periodicity of the sampling time sequence, $A(k), C(k)$ are periodic matrices. FD systems can be designed for the MSD system based on the time-varying model (126).

\subsubsection{Parity-relation-based FD scheme for MSD systems}

The input-output relationship of (126) over the moving horizon $\left[t_{k-s}, t_{k}\right]$, where $s$ denotes the length of the moving horizon, is

$$
\widetilde{\psi}(k)=H_{o, s, k} x(k-s)+H_{s, k}(\tilde{u}(k)+\tilde{d}(k)+\tilde{f}(k)),
$$

where $\tilde{\psi}(k), \tilde{u}(k), \tilde{d}(k)$, and $\tilde{f}(k)$ are stacked vectors based on $\bar{\psi}(j), \bar{u}(j), \bar{d}(j)$, and $\bar{f}(j), j=k-s, \ldots, k$, respectively, $H_{o, s, k}$ is constructed according to (83),

$$
H_{s, k}=\left[\begin{array}{ccc}
O & O & \cdots \\
C(k-s+1) & \ddots & \ddots \\
\vdots & \ddots & O \\
C(k) A(k-1) \cdots A(k-s+1) & \cdots & C(k)
\end{array}\right] .
$$

Build a parity-relation-based residual generator with

$$
r(k)=V_{k}\left(\tilde{\psi}(k)-H_{s, k} \tilde{u}(k)\right), \quad V_{k} H_{o, s, k}=0,
$$

where $V_{k}$ is a periodically time-varying parity matrix (or vector). To describe the influence of continuous-time signals $d(t), f(t)$ on the multirate-sampled outputs, linear timevarying operators $\Psi_{k}^{\delta}$ ( $\delta$ standing for $d$ or $\left.f\right)$,

$$
\tilde{\delta}(k)=\Psi_{k}^{\delta} \delta_{k}(t)=\left[\begin{array}{c}
\int_{t_{k-s+1}}^{t_{k s}} e^{A_{c}\left(t_{k-s+1}-t\right)} E_{\delta c} \delta(t) d t \\
\int_{t_{k-s+1}}^{k_{-s+1}} e^{A_{c}\left(t_{k-s+2}-t\right)} E_{\delta c} \delta(t) d t \\
\vdots \\
\int_{t_{k-1}}^{t_{k}} e^{A_{c}\left(t_{k}-t\right)} E_{\delta c} \delta(t) d t
\end{array}\right],
$$

are introduced and the residual dynamics is described by

$$
r(k)=V_{k} H_{s, k}(\tilde{d}(k)+\tilde{f}(k))=V_{k} H_{s, k}\left(\Psi_{k}^{d} d_{k}(t)+\Psi_{k}^{f} f_{k}(t)\right) .
$$

The optimal selection of $V_{k}$ can be formulated similar to (117) with $\Psi^{d}\left(\Psi^{d}\right)^{*}$ and $\Psi^{f}\left(\Psi^{f}\right)^{*}$ substituted by $\Psi_{k}^{d}\left(\Psi_{k}^{d}\right)^{*}$ and $\Psi_{k}^{f}\left(\Psi_{k}^{f}\right)^{*}$, respectively. Using the same technique, $\Psi_{k}^{\delta}\left(\Psi_{k}^{\delta}\right) *$ are derived to be

$$
\begin{aligned}
\Psi_{k}^{\delta}\left(\Psi_{k}^{\delta}\right)^{*} & =\operatorname{diag}\left\{\Psi_{k, s}^{\delta}, \ldots, \Psi_{k, 1}^{\delta}\right\}, \\
\Psi_{k, j}^{\delta} & =\int_{0}^{t_{k-j+1}-t_{k-j}} e^{A_{c} t} E_{\delta c} E_{\delta c}^{T} e^{A_{c}^{T} t} d t, \quad j=s, \ldots, 1 .
\end{aligned}
$$

Due to the periodicity of $\Psi_{k}^{\delta}\left(\Psi_{k}^{\delta}\right)$, the optimization problem needs to be solved over one period.

\subsubsection{Observer-based FD scheme for MSD systems}

For the aim of fault detection, a fast rate time-varying observer-based residual generator can be constructed as

$$
\begin{aligned}
\hat{x}(k+1) & =A(k) \hat{x}(k)+\bar{u}(k)+L(k)(\bar{\psi}(k)-\hat{\psi}(k)), \\
r(k) & =W(k)(\bar{\psi}(k)-\hat{\psi}(k)), \quad \hat{\psi}(k)=C(k) \widehat{x}(k),
\end{aligned}
$$

where the gain matrix $L(k)$ and the weighting matrix $W(k)$ are time-varying matrices to be determined. The dimensions of $L(k)$ and $W(k)$ may change with the number of available sampled output signals. Considering the periodicity of the matrices $A(k), C(k),(134)$ can be designed as a periodic observer. Define the state estimation error as $e(k)=x(k)-\hat{x}(k)$. The dynamics of residual generator (134) is governed by

$$
\begin{aligned}
e(k+1) & =(A(k)-L(k) C(k)) e(k)+\bar{d}(k)+\bar{f}(k), \\
r(k) & =W(k) C(k) e(k) .
\end{aligned}
$$

Introduce linear time-varying operators $\Gamma_{k}^{\delta}(\delta$ standing for $d$ or $f$ ),

$$
\Gamma_{k}^{\delta} \delta_{k}(t)=\int_{t_{k}}^{t_{k+1}} e^{A_{c}\left(t_{k+1}-t\right)} E_{\delta c} \delta(t) d t
$$

to rewrite the residual dynamics as

$$
\begin{aligned}
e(k+1) & =(A(k)-L(k) C(k)) e(k)+\Gamma_{k}^{d} d_{k}(t)+\Gamma_{k}^{f} f_{k}(t), \\
r(k) & =W(k) C(k) e(k) .
\end{aligned}
$$


To enhance the robustness of the FD system to the unknown disturbances without loss of the sensitivity to the faults, the design problem is formulated as

$$
\begin{aligned}
& \sup _{L(k), W(k)} J_{\mathrm{MSD}, \mathrm{OBS}, \infty / \infty}(L(k), W(k)) \\
&= \sup _{L(k), W(k)} \frac{\sup _{d=0, f \in \mathcal{L}_{2}-\{0\}}\left(\|r\|_{2} /\|f\|_{2}\right)}{\sup _{f=0, d \in \mathscr{L}_{2}-\{0\}}\left(\|r\|_{2} /\|d\|_{2}\right)}, \\
& \sup _{L(k), W(k)} J_{\mathrm{MSD}, \mathrm{OBS},-/ \infty}(L(k), W(k)) \\
&=\sup _{L(k), W(k)} \frac{\inf _{d=0, f \in \mathscr{L}_{2}-\{0\}}\left(\|r\|_{2} /\|f\|_{2}\right)}{\sup _{f=0, d \in \mathcal{L}_{2}-\{0\}}\left(\|r\|_{2} /\|d\|_{2}\right)} .
\end{aligned}
$$

By analyzing $\Gamma_{k}^{\delta}\left(\Gamma_{k}^{\delta}\right)^{*}$, optimization problems (138) are transformed into equivalent optimization problems of discrete LTP system

$$
\begin{aligned}
e(k+1) & =(A(k)-L(k) C(k)) e(k)+\bar{E}_{d}(k) d_{\mathrm{eq}}(k)+\bar{E}_{f}(k) f_{\mathrm{eq}}(k), \\
r(k) & =W(k) C(k) e(k),
\end{aligned}
$$

where the $l_{2}$-norms of $d_{\mathrm{eq}}(k)$ and $f_{\mathrm{eq}}(k)$ in (139) have the same upper bounds, respectively, with the $\mathcal{L}_{2}$-norms of $d(t)$ and $f(t)$ in (104), the matrices $\bar{E}_{d}(k)$ and $\bar{E}_{f}(k)$ are timevarying matrices reflecting the sampling effect and satisfy

$$
\bar{E}_{\delta}(k) \bar{E}_{\delta}^{T}(k)=\int_{0}^{t_{k+1}-t_{k}} e^{A_{c} t} E_{\delta c} E_{\delta c}^{T} e^{A_{c}^{T} t} d t .
$$

Then, optimization problems (138) can be solved with the approaches introduced in Section 3.2.2.

\subsection{FD of NSD systems}

The same design procedures introduced in the last subsection can be applied to the FD of NSD systems by reordering the sampling instants. The main difference lies in that in general NSD systems, $A(k), C(k), \bar{E}_{d}(k), \bar{E}_{f}(k)$ are time-varying matrices but not periodic matrices. In consequence, for the NSD systems

(i) if the parity space approach is used, then the timevarying parity matrix $V(k)$ needs to be calculated at each time instant,

(ii) if the observer-based approach is adopted, then the observer gain matrix $L(k)$ needs to guarantee the stability of the resulting linear time-varying system.

\subsection{Influence of sampling period on optimal FD performance}

Sampling period is an important parameter in SD systems. Recently, the influence of the sampling period on the optimal FD performance has been investigated in [70, 71]. Suppose that for a given continuous-time process (104) three different sampling schemes are considered: single-rate sampling with sampling period $h$, single-rate sampling with sampling period $\rho h$, multirate sampling with base period $h$ and system period $\rho$, where $\rho \geq 1$ is an integer. It is proven that the optimal performance indeces are related by

$$
\begin{gathered}
J_{\mathrm{SSD}, \mathrm{OBS}, \infty / \infty, h} \geq J_{\mathrm{MSD}, \mathrm{OBS}, \infty / \infty} \geq J_{\mathrm{SSD}, \mathrm{OBS}, \infty / \infty, \rho h,}, \\
J_{\mathrm{SSD}, \mathrm{PS}, \infty / \infty, h} \geq J_{\mathrm{MSD}, \mathrm{PS}, \infty / \infty} \geq J_{\mathrm{SSD}, \mathrm{PS}, \infty / \infty, \rho h .}
\end{gathered}
$$

That means that, with the increase of the sampling period, the FD performance will become worse. It can be intuitively interpreted as the consequence of information reduction caused by the increase of the sampling period. However, we would like to emphasize that such a conclusion does not hold for all performance indices, for instance, the $H_{-} / H_{\infty}$ index.

\section{CONCLUDING REMARKS}

In this paper, standard methods for FD in discrete LTI systems have been reviewed and recent development in FD for discrete LTP and SD systems has been summarized. In case of discrete LTI systems, the basic idea, full decoupling and optimization problems, and the corresponding solutions are introduced. It can be seen that different FD approaches are closely related to each other. The FD problem of discrete LTP systems can be handled either indirectly by lifting or directly by considering the periodicity of the system matrices. In SD systems the main problem is to take into account the intersample behavior and to develop direct FD approaches. With the aid of operators, the FD problem of SSD, MSD, and NSD systems can be transformed, respectively, into the FD problem of discrete LTI, LTP, and linear time-varying systems. The methods introduced in this paper have found several interesting applications in the emerging research area of embedded networked control systems (emNCS) [72, 73]. Because of the limited data rate, the sampling mechanisms become an important design parameter in emNCS and have decisive influence on the real-time network and computing performance and FD performance.

\section{REFERENCES}

[1] M. Basseville and I. Nikiforov, Detection of Abrupt Changes Theory and Application, Prentice-Hall, Englewood Cliffs, NJ, USA, 1993.

[2] J. Gertler, Fault Detection and Diagnosis in Engineering Systems, Marcel Dekker, New York, NY, USA, 1998.

[3] R. Mangoubi, Robust Estimation and Failure Detection, Springer, New York, NY, USA, 1998.

[4] J. Chen and R. J. Patton, Robust Model-Based Fault Diagnosis for Dynamic Systems, Kluwer Academic Publishers, Boston, Mass, USA, 1999.

[5] R. J. Patton, P. M. Frank, and R. N. Clark, Issues of Fault Diagnosis for Dynamic Systems, Springer, London, UK, 2000.

[6] M. Blanke, M. Kinnaert, J. Lunze, and M. Staroswiecki, Diagnosis and Fault-Tolerant Control, Springer, New York, NY, USA, 2003.

[7] P. M. Frank, S. X. Ding, and T. Marcu, "Model-based fault diagnosis in technical processes," Transactions of the Institute of Measurement and Control, vol. 22, no. 1, pp. 57-101, 2000.

[8] E. Y. Chow and A. S. Willsky, "Analytical redundancy and the design of robust failure detection systems," IEEE Transactions on Automatic Control, vol. 29, pp. 603-614, 1984. 
[9] H. Ishii and B. Francis, Limited Data Rate in Control Systems with Networks, Springer, Berlin, Germany, 2002.

[10] K. Zhou, Essential of Robust Control, Prentice-Hall, Englewood Cliffs, NJ, USA, 1998.

[11] J. Wuennenberg, Observer-Based Fault Detection in Dynamic Systems, Ph.D. thesis, University of Duisburg, Duisburg, Germany, 1990.

[12] S. X. Ding, E. L. Ding, T. Jeinsch, and P. Zhang, "An approach to a unified design of FDI systems," in Proceedings of the $3 \mathrm{rd}$ Asian Control Conference, pp. 2812-2817, Shanghai, China, July 2000.

[13] S. X. Ding, E. L. Ding, and T. Jeinsch, "A numerical approach to optimization of FDI systems," in Proceedings of the 37th IEEE Conference on Decision and Control (CDC'98), pp. 11371142, Tampa, Fla, USA, December 1998.

[14] S. X. Ding, E. L. Ding, and T. Jeinsch, "An approach to analysis and design of observer and parity relation based FDI systems," in Proceedings of the 14th Triennial World Congress of the International Federation of Automatic Control, pp. 37-42, Beijing, China, July 1999.

[15] S. Schneider, N. Weinhold, S. X. Ding, and A. Rehm, "Parity space based FDI-scheme for vehicle lateral dynamics," in Proceedings of the IEEE International Conference on Control Applications (CCA '05), pp. 1409-1414, Toronto, Canada, August 2005.

[16] X. Ding, L. Guo, and T. Jeinsch, "A characterization of parity space and its application to robust fault detection," IEEE Transactions on Automatic Control, vol. 44, no. 2, pp. 337-343, 1999.

[17] H. Ye, G. Z. Wang, S. X. Ding, and H. Y. Su, "An IIR filter based parity space approach for fault detection," in Proceedings of the 15th Triennial World Congress of the International Federation of Automatic Control, Barcelona, Spain, July 2002.

[18] P. Zhang and S. X. Ding, "Multi-objective design of robust fault detection systems," in Proceedings of the 6th IFAC Symposium on Fault Detection, Supervision and Safety of Technical Processes (SAFEPROCESS '06), pp. 1453-1458, Beijing, China, August-September 2006.

[19] S. X. Ding, P. Zhang, B. Huang, E. L. Ding, and P. M. Frank, "An approach to norm and statistical methods based residual evaluation," in Proceedings of the 10th IEEE International Conference on Methods and Models in Automation and Robotics (MMAR '04), pp. 777-780, Miedzyzdroje, Poland, AugustSeptember 2004.

[20] X. Ding and P. M. Frank, "Fault detection via factorization approach," Systems and Control Letters, vol. 14, no. 5, pp. 431436, 1990.

[21] X. Ding and P. M. Frank, "Fault detection via optimally robust detection filters," in Proceedings of the 28th Conference on Decision and Control (CDC '89), vol. 2, pp. 1767-1772, Tampa, Fla, USA, December 1989.

[22] S. X. Ding, T. Jeinsch, P. M. Frank, and E. L. Ding, "A unified approach to the optimization of fault detection systems," International Journal of Adaptive Control and Signal Processing, vol. 14, no. 7, pp. 725-745, 2000.

[23] P. Zhang, S. X. Ding, G. Z. Wang, and D. H. Zhou, "An approach to fault detection of sampled-data systems," Tech. Rep., submitted to IEEE Transactions on Automatic Control.

[24] P. Zhang, S. X. Ding, G. Z. Wang, and D. H. Zhou, "Fault detection of linear discrete-time periodic systems," IEEE Transactions on Automatic Control, vol. 50, no. 2, pp. 239-244, 2005.

[25] N. Liu and K. Zhou, "Optimal and analytic solutions to robust fault detection problems," submitted to IEEE Transactions on Automatic Control.
[26] P. Zhang, S. X. Ding, G. Z. Wang, D. H. Zhou, and E. L. Ding, "An $H_{\infty}$ approach to fault detection for sampled-data systems," in Proceedings of the American Control Conference (ACC '02), vol. 3, pp. 2196-2201, Anchorage, Alaska, USA, May 2002.

[27] P. Zhang, H. Ye, S. X. Ding, G. Z. Wang, and D. H. Zhou, "On the relationship between parity space and $H_{2}$ approaches to fault detection," Systems and Control Letters, vol. 55, no. 2, pp. 94-100, 2006.

[28] H. Ye, S. X. Ding, and G. Z. Wang, "Integrated design of fault detection systems in time-frequency domain," IEEE Transactions on Automatic Control, vol. 47, no. 2, pp. 384-390, 2002.

[29] H. Ye, G. Z. Wang, and S. X. Ding, "A new parity space approach for fault detection based on stationary wavelet transform," IEEE Transactions on Automatic Control, vol. 49, no. 2, pp. 281-287, 2004.

[30] S. X. Ding, P. M. Frank, E. L. Ding, and T. Jeinsch, "Fault detection system design based on a new trade-off strategy," in Proceedings of the 39th IEEE Conference on Decision and Control (CDC '00), vol. 4, pp. 4144-4149, Sydney, Australia, December 2000.

[31] P. M. Frank and X. Ding, "Frequency domain approach to optimally robust residual generation and evaluation for modelbased fault diagnosis," Automatica, vol. 30, no. 5, pp. 789-904, 1994.

[32] P. Zhang, S. X. Ding, G. Z. Wang, and D. H. Zhou, "Fault detection for linear discrete-time periodic systems," in Proceedings of the 5th IFAC Symposium on Fault Detection, Supervision and Safety of Technical Processes (SAFEPROCESS '03), pp. 247252, Washington, DC, USA, 2003.

[33] M. Hou and R. J. Patton, "An LMI approach to $H_{-} / H_{\infty}$ fault detection observers," in Proceedings of the UKACC International Conference on Control, vol. 1, pp. 305-310, Exeter, UK, September 1996.

[34] F. Rambeaux, F. Hamelin, and D. Sauter, "Robust residual generation via LMI," in Proceedings of the 14th Triennial World Congress of the International Federation of Automatic Control, pp. 240-246, Beijing, China, July 1999.

[35] D. Henry and A. Zolghadri, "Design and analysis of robust residual generators for systems under feedback control," $A u$ tomatica, vol. 41, no. 2, pp. 251-264, 2005.

[36] J. Liu, J. L. Wang, and G.-H. Yang, "An LMI approach to minimum sensitivity analysis with application to fault detection," Automatica, vol. 41, no. 11, pp. 1995-2004, 2005.

[37] R. H. Chen, D. L. Mingori, and J. L. Speyer, "Optimal stochastic fault detection filter," Automatica, vol. 39, no. 3, pp. 377390, 2003.

[38] S. Bittanti and P. Colaneri, "Analysis of discrete-time linear periodic systems," Control and Dynamics Systems, vol. 78, pp. 313-339, 1996.

[39] P. Colaneri and V. Kucera, "The model matching problem for periodic discrete-time systems," IEEE Transactions on Automatic Control, vol. 42, no. 10, pp. 1472-1476, 1997.

[40] B. P. Lampe, M. A. Obraztsov, and E. N. Rosenwasser, "Statistical analysis of stable FDLCP systems by parametric transfer matrices," International Journal of Control, vol. 78, no. 10, pp. 747-761, 2005.

[41] S. Bittanti and P. Colaneri, "Periodic Control," in John Wiley Encyclopaedia on Electrical and Electronic Engineering, J. Webster, Ed., vol. 16, pp. 240-253, John Wiley \& Sons, New York, NY, USA, 1999.

[42] S. Bittanti and P. Colaneri, "Invariant representations of discrete-time periodic systems," Automatica, vol. 36, no. 12, pp. 1777-1793, 2000. 
[43] M. S. Fadali and S. Gummuluri, "Robust observer-based fault detection for periodic systems," in Proceedings of the American Control Conference (ACC '01), vol. 1, pp. 464-469, Arlington, Va, USA, June 2001.

[44] A. Varga, "Design of fault detection filters for periodic systems," in Proceedings of the 43rd IEEE Conference on Decision and Control (CDC '04), pp. 4800-4805, Atlantis, Bahamas, December 2004.

[45] P. Zhang, S. X. Ding, and T. Jeinsch, "Study on full decoupling problem of linear periodic systems," in Proceedings of the 16th Triennial World Congress of the International Federation of Automatic Control, Prague, Czech Republic, July 2005.

[46] T. W. Chen and B. Francis, Optimal Sampled-Data Control Systems, Springer, London, UK, 1995.

[47] E. Rosenwasser and B. P. Lampe, Computer Controlled Systems-Analysis and Design with Process-Orientated Models, Springer, London, UK, 2000.

[48] G. M. Kranc, "Input-output analysis of multirate feedback systems," IRE Transactions on Automatic Control, vol. 3, no. 1, pp. 21-28, 1957.

[49] R. E. Kalman and J. E. Bertram, "A unified approach to the theory of sampling systems," Journal of the Franklin Institute, vol. 267, no. 5, pp. 405-436, 1959.

[50] R. A. Meyer and C. S. Burrus, "A unified analysis of multirate and periodically time-varying digital filters," IEEE Transactions on Circuits and Systems, vol. 22, no. 3, pp. 162-168, 1975.

[51] M. Araki and T. Hagiwara, "Pole assignment by multirate sampled-data output feedback," International Journal of Control, vol. 44, no. 6, pp. 1661-1673, 1986.

[52] L. Qiu and T. W. Chen, "Multirate sampled-data systems: all $H_{\infty}$ suboptimal controllers and the minimum entropy controller," IEEE Transactions on Automatic Control, vol. 44, no. 3 , pp. 537-550, 1999.

[53] S. Lall and G. Dullerud, "An LMI solution to the robust synthesis problem for multi-rate sampled-data systems," Automatica, vol. 37, no. 12, pp. 1909-1922, 2001.

[54] J. Sheng, T. W. Chen, and S. L. Shah, "Generalized predictive control for non-uniformly sampled systems," Journal of Process Control, vol. 12, no. 8, pp. 875-885, 2002.

[55] G. Kreisselmeier, "On sampling without loss of observability/controllability," IEEE Transactions on Automatic Control, vol. 44, no. 5, pp. 1021-1025, 1999.

[56] W. Li, S. L. Shah, and D. Y. Xiao, "Kalman filters for nonuniformly sampled multirate systems," in Proceedings of the 16th Triennial World Congress of the International Federation of Automatic Control, Prague, Czech Republic, July 2005.

[57] W. Li, Z. Han, and S. L. Shah, "Subspace identification for FDI in systems with non-uniformly sampled multirate data," $\mathrm{Au}$ tomatica, vol. 42, no. 4, pp. 619-627, 2006.

[58] P. Zhang, S. X. Ding, G. Z. Wang, and D. H. Zhou, "An FDI approach for sampled-data systems," in Proceedings of the American Control Conference (ACC '01), vol. 4, pp. 2702-2707, Arlington, Va, USA, June 2001.

[59] P. Zhang, S. X. Ding, G. Z. Wang, and D. H. Zhou, "A frequency domain approach to fault detection in sampled-data systems," Automatica, vol. 39, no. 7, pp. 1303-1307, 2003.

[60] P. Zhang, S. X. Ding, G. Z. Wang, and D. H. Zhou, "An approach to fault detection of sampled-data systems," Tech. Rep., Institute of Automatic Control and Complex Systems(AKS), University of Duisburg, Duisburg, Germany, 2003.

[61] N. Viswanadham and K. D. Minto, "Fault diagnosis in multirate sampled data systems," in Proceedings of the 29th IEEE
Conference on Decision and Control (CDC '90), vol. 6, pp. 3666-3671, Honolulu, Hawaii, USA, December 1990.

[62] M. S. Fadali and W. Liu, "Fault detection for systems with multirate sampling," in Proceedings of the American Control Conference (ACC'98), pp. 3302-3306, Philadelphia, Pa, USA, June 1998.

[63] M. S. Fadali and W. Liu, "Observer-based robust fault detection for a class of multirate sampled-data linear systems," in Proceedings of the American Control Conference (ACC '99), vol. 1, pp. 97-98, San Diego, Calif, USA, June 1999.

[64] M. S. Fadali and H. E. Emara-Shabaik, "Robust fault detection for a class of multirate linear systems," in Proceedings of the American Control Conference (ACC '00), vol. 2, pp. 1210-1214, Chicago, Ill, USA, June 2000.

[65] P. Zhang, S. X. Ding, G. Z. Wang, and D. H. Zhou, "Fault detection for multirate sampled-data systems with time delays," International Journal of Control, vol. 75, no. 18, pp. 1457-1471, 2002.

[66] P. Zhang, S. X. Ding, G. Z. Wang, and D. H. Zhou, “Observerbased approaches to fault detection in multirate sampled-data systems," in Proceedings of the 4th Asian Control Conference, pp. 1367-1372, Singapore, September 2002.

[67] I. Izadi, Q. Zhao, and T. W. Chen, "An optimal scheme for fast rate fault detection based on multirate sampled data," Journal of Process Control, vol. 15, no. 3, pp. 307-319, 2005.

[68] I. Izadi, Q. Zhao, and T. W. Chen, "An $H_{\infty}$ approach to fast rate fault detection for multirate sampled-data systems," Journal of Process Control, vol. 16, no. 6, pp. 651-658, 2006.

[69] P. Zhang and S. X. Ding, "Fault detection of multirate sampled-data systems based on periodic system theory," in Proceedings of the European Control Conference, Kos, Greece, July 2007.

[70] P. Zhang, S. X. Ding, R. J. Patton, and C. Kambhampati, "Adaptive and cooperative sampling in networked control systems," in Proceedings of the IEEE International Conference on Networking, Sensing and Control (ICNSC '07), pp. 398-403, London, UK, April 2007.

[71] P. Zhang and S. X. Ding, "On monotonicity of a class of optimal fault detection performance vs. sampling period," in Proceedings of the 46th IEEE Conference on Decision and Control (CDC'07), New Orleans, La, USA, December 2007.

[72] S. X. Ding, P. Zhang, and E. L. Ding, "Observer-based monitoring of distributed net-worked control systems," in Proceedings of the 6th IFAC Symposium on Fault Detection, Supervision and Safety of Technical Processes (SAFEPROCESS '06), pp. 337342, Beijing, China, August-September 2006.

[73] P. Zhang and S. X. Ding, "Fault detection of networked control systems with limited communication," in Proceedings of the 6th IFAC Symposium on Fault Detection, Supervision and Safety of Technical Processes (SAFEPROCESS '06), pp. 1135-1140, Beijing, China, August-September 2006. 

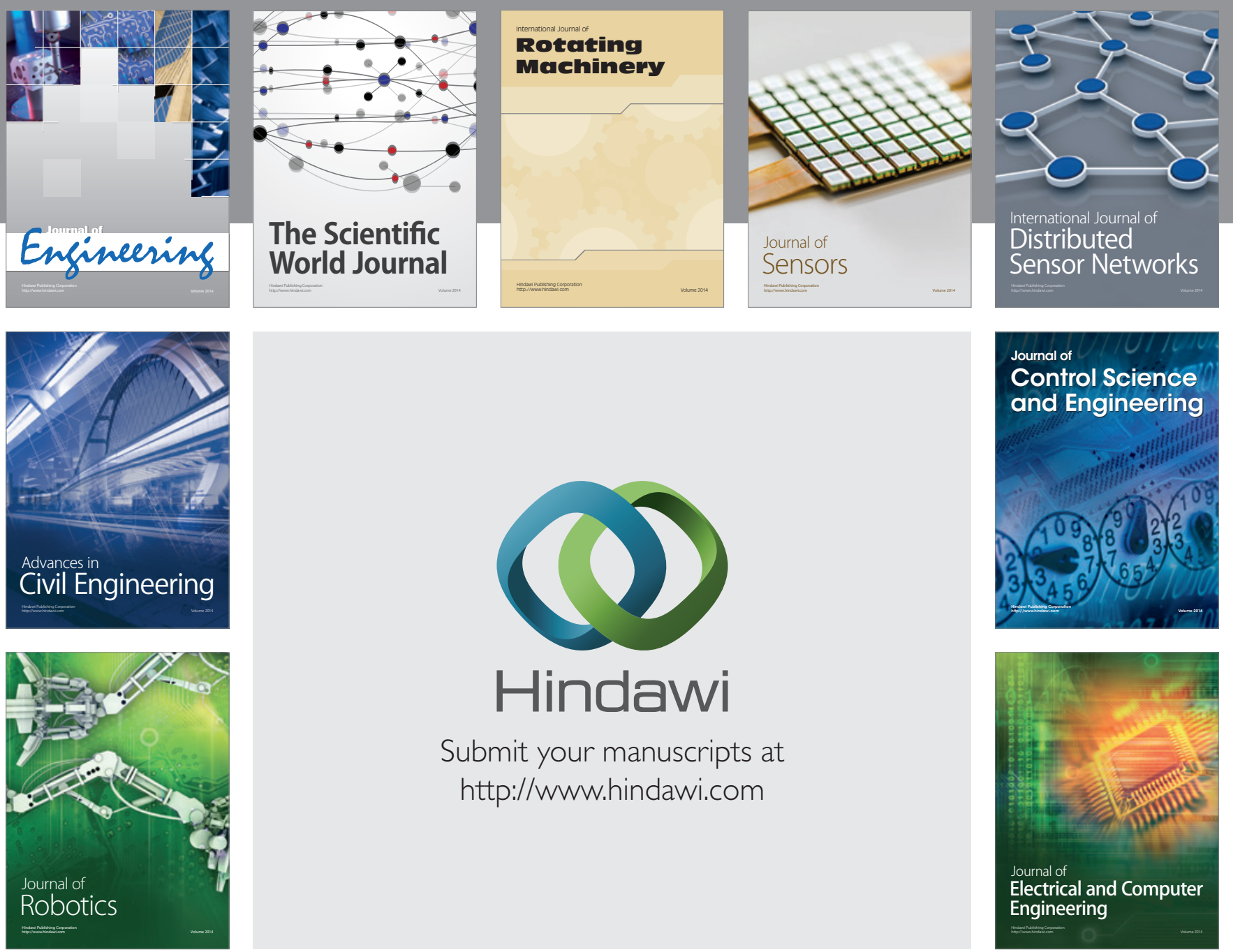

Submit your manuscripts at

http://www.hindawi.com
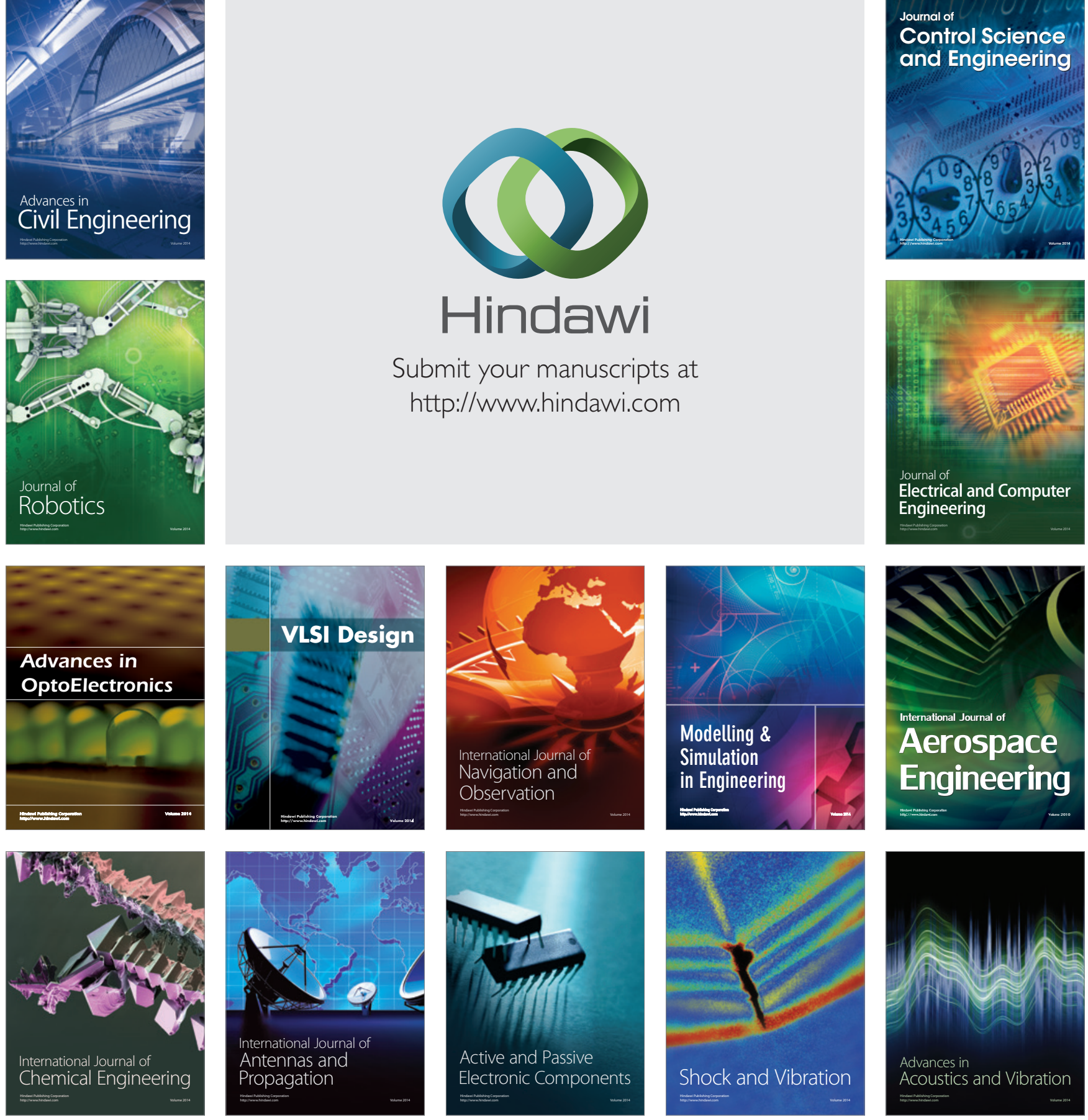